\title{
PHASE TRANSITIONS FOR THE BRUSSELATOR MODEL
}

\author{
TIAN MA AND SHOUHONG WANG
}

\begin{abstract}
Dynamic phase transitions of the Brusselator model is carefully analyzed, leading to a rigorous characterization of the types and structure of the phase transitions of the model from basic homogeneous states. The study is based on the dynamic transition theory developed recently by the authors.
\end{abstract}

\section{INTRODUCTION}

Belousov-Zhabotinsky (BZ) reactions are now one of a class of reactions that serve as a classical example of non-equilibrium thermodynamics, resulting in the establishment of a nonlinear chemical oscillator.

The main objective of this article is to study the dynamic phase transitions of the Belousov-Zhabotinsky reactions focusing on the Brusselator, first introduced by 11. The Brusselator is one of the simplest models in nonlinear chemical systems. It has six components, four of which retain constants, and the other two permit their concentrations vary with time and space. The chemical reaction consists of four irreversible steps, given by

$$
\begin{aligned}
& \mathrm{A} \stackrel{k_{1}}{\longrightarrow} \mathrm{X}, \\
& \mathrm{B}+\mathrm{X} \stackrel{k_{2}}{\longrightarrow} \mathrm{Y}+\mathrm{D}, \\
& 2 \mathrm{X}+\mathrm{Y} \stackrel{k_{3}}{\longrightarrow} 3 \mathrm{X}, \\
& \mathrm{X} \stackrel{k_{4}}{\longrightarrow} \mathrm{E} .
\end{aligned}
$$

where $A$ and $B$ are constant components, $D$ and $E$ are products, and $X$ and $Y$ are the two components variable in time and space. Over the years, there have been extensive studies for the Brusselator and related chemical reaction problems; see among many others [13, 2, 1, 4, 12, and the references therein.

In this article, we address the dynamic phase transition of the Brusselator model. In particular, we derive a complete characterization of the transition from the homogeneous state. There are two aspects of this characterization. First our analysis shows that both the transitions to multiple equilibria and to time-periodic solutions (spatiotemporal oscillations) can occur for the Brusselator model, and are precisely determined by the sign of an explicit nondimensional parameter $\delta_{0}-\delta_{1}$ as defined by (3.5) and (3.6).

Then in both transition cases, the dynamic behavior of the transition is classified based on the new dynamical classification scheme, introduced as part of the

1991 Mathematics Subject Classification. 80A30, 35Q92.

Key words and phrases. Belousov-Zhabotinsky chemical reactions, Brusselator, dynamic phase transition, spatiotemporal oscillations.

The work was supported in part by grants from the Office of Naval Research, the National Science Foundation, and the National Science Foundation of China. 
dynamical transition theory developed recently by the authors; see [5, 6, 7]. With this classification scheme, phase transitions are classified into three types: Type-I, Type-II and Type-III, which, in more mathematically intuitive terms, are called continuous, jump and mixed transitions respectively. For the Brusselator, the distinction of the dynamic transition types are determined, again, by the signs of some nondimensional computable parameters.

It is worth mentioning that the main philosophy of the dynamic transition theory is to search for the full set of transition states, giving a complete characterization on stability and transition. The set of transition states is represented by a local attractor. Following this philosophy, the dynamic transition theory is developed to identify the transition states and to classify them both dynamically and physically. With this theory, many long standing phase transition problems are either solved or become more accessible, providing new insights to both theoretical and experimental studies for the underlying physical problems.

This article is organized as follows. Section 2 introduces the model and its mathematical set-up, and Section 3 addresses the principle of exchange of stabilities. Dynamic transitions of the model are addressed in Sections 4-6, with physical remarks of the main results given in Section 7 .

\section{The Model and its Mathematical Set-up}

Let $u_{1}, u_{2}, a$ and $b$ stand for the concentrations of $X, Y, A$ and $B$. Then the reaction equations of (1.1) read

$$
\begin{aligned}
\frac{\partial u_{1}}{\partial t} & =\sigma_{1} \Delta u_{1}+k_{1} a-\left(k_{2} b+k_{4}\right) u_{1}+k_{3} u_{1}^{2} u_{2}, \\
\frac{\partial u_{2}}{\partial t} & =\sigma_{2} \Delta u_{2}+k_{2} b u_{1}-k_{3} u_{1}^{2} u_{2} .
\end{aligned}
$$

To get the nondimensional form of (2.1), let

$$
\begin{array}{lll}
t=k_{4}^{-1} t^{\prime}, & x=l x^{\prime}, & u_{i}=\left(\frac{k_{4}}{k_{3}}\right)^{1 / 2} u_{i}^{\prime}, \\
a=\left(k_{4}^{3} / k_{1}^{2} k_{3}\right)^{1 / 2} \alpha, & b=\left(k_{4} / k_{2}\right) \lambda, & \sigma_{i}=l^{2} k_{4} \mu_{i},
\end{array}
$$

for $i=1,2$. Omitting the primes, the equations (2.1) become

$$
\begin{aligned}
& \frac{\partial u_{1}}{\partial t}=\mu_{1} \Delta u_{1}+\alpha-(\lambda+1) u_{1}+u_{1}^{2} u_{2}, \\
& \frac{\partial u_{2}}{\partial t}=\mu_{2} \Delta u_{2}+\lambda u_{1}-u_{1}^{2} u_{2},
\end{aligned}
$$

where $u_{1}, u_{2} \geq 0, \Omega \subset \mathbb{R}^{n}(1 \leq n \leq 3)$ is a bounded domain, and

$$
\mu_{1}, \mu_{2}, \alpha, \lambda>0 \text {. }
$$

The equations (2.2) have a constant steady state solution

$$
u_{0}=(\alpha, \lambda / \alpha) \text {. }
$$

Make the translation

$$
u_{1}=\alpha+v_{1}, \quad u_{2}=\frac{\lambda}{\alpha}+v_{2},
$$


then the equations (2.2) are written as

$$
\begin{aligned}
& \frac{\partial v_{1}}{\partial t}=\mu_{1} \Delta v_{1}+(\lambda-1) v_{1}+\alpha^{2} v_{2}+\frac{2 \lambda}{\alpha} v_{1}^{2}+2 \alpha v_{1} v_{2}+v_{1}^{2} v_{2}, \\
& \frac{\partial v_{2}}{\partial t}=\mu_{2} \Delta v_{2}-\lambda v_{1}-\alpha^{2} v_{2}-\frac{2 \lambda}{\alpha} v_{1}^{2}-2 \alpha v_{1} v_{2}-v_{1}^{2} v_{2} .
\end{aligned}
$$

There are two types of physically-sound boundary conditions: the Dirichlet boundary condition

$$
v=\left(v_{1}, v_{2}\right)=0 \quad \text { on } \partial \Omega,
$$

and the Neumann boundary condition

$$
\frac{\partial v}{\partial n}=0 \quad \text { on } \partial \Omega
$$

Define the function spaces

$$
\begin{aligned}
& H=L^{2}\left(\Omega, \mathbb{R}^{2}\right), \\
& H_{1}= \begin{cases}H^{2}\left(\Omega, \mathbb{R}^{2}\right) \cap H_{0}^{1}\left(\Omega, \mathbb{R}^{2}\right) & \text { for b.c (2.4) }, \\
\left\{v \in H^{2}\left(\Omega, \mathbb{R}^{2}\right)\left|\frac{\partial v}{\partial n}\right|_{\partial \Omega}=0\right\} & \text { for b.c. (2.5). }\end{cases}
\end{aligned}
$$

Define the operators $L_{\lambda}=A+B_{\lambda}$ and $G: H_{1} \rightarrow H$ by

$$
\begin{aligned}
& A v=\left(\mu_{1} \Delta v_{1}, \mu_{2} \Delta v_{2}\right), \\
& B_{\lambda} v=\left((\lambda-1) v_{1}+\alpha^{2} v_{2},-\lambda v_{1}-\alpha^{2} v_{2}\right), \\
& G v=\left(\frac{2 \lambda}{\alpha} v_{1}^{2}+2 \alpha v_{1} v_{2}+v_{1}^{2} v_{2},-\frac{2 \lambda}{\alpha} v_{1}^{2}-2 \alpha v_{1} v_{2}-v_{1}^{2} v_{2}\right) .
\end{aligned}
$$

Thus the equations (2.3) with (2.4) or with (2.5) can be written in the following abstract form

$$
\frac{d v}{d t}=L_{\lambda} v+G(v, \lambda)
$$

\section{Principle of Exchange of Stability (PES)}

Consider the eigenvalue quations of (2.3)

$$
\begin{aligned}
& \mu_{1} \Delta v_{1}+(\lambda-1) v_{1}+\alpha^{2} v_{2}=\beta v_{1}, \\
& \mu_{2} \Delta v_{2}-\lambda v_{1}-\alpha^{2} v_{2}=\beta v_{2},
\end{aligned}
$$

with the boundary condition (2.4) or 2.5).

Let $\rho_{k}$ and $e_{k}$ be the $k$ th eigenvalue and eigenvector of the Laplacian with either the Dirichlet or the Neumann condition:

$$
\begin{aligned}
& \Delta e_{k}=-\rho_{k} e_{k}, \\
& \left.e_{k}\right|_{\partial \Omega}=0 \quad \text { or }\left.\frac{\partial e_{k}}{\partial n}\right|_{\partial \Omega}=0 .
\end{aligned}
$$

Denote by $M_{k}$ the matrix given by

$$
M_{k}=\left(\begin{array}{cc}
-\mu_{1} \rho_{k}+\lambda-1 & \alpha^{2} \\
-\lambda & -\mu_{2} \rho_{k}-\alpha^{2}
\end{array}\right), \quad k=1,2, \cdots .
$$


It is clear that all eigenvalues $\beta_{k}^{ \pm}$and eigenvectors $\phi_{k}^{ \pm}$of (3.1) satisfy the following equations

$$
\begin{aligned}
& \phi_{k}^{ \pm}=\xi_{k}^{ \pm} e_{k}, \\
& M_{k} \xi_{k}^{ \pm}=\beta_{k}^{ \pm} \xi_{k}^{ \pm},
\end{aligned}
$$

where $\xi_{k}^{ \pm} \in \mathbb{R}^{2}$ are the eigenvectors of $M_{k}, \beta_{k}^{ \pm}$are the eigenvalues of $M_{k}$, which are expressed as

$$
\begin{aligned}
\beta_{k}^{ \pm}(\lambda)= & \frac{1}{2}\left[\lambda-\left(\mu_{1} \rho_{k}+\mu_{2} \rho_{k}+\alpha^{2}+1\right)\right] \\
& \pm \frac{1}{2}\left[\left(\lambda-\mu_{1} \rho_{k}-\mu_{2} \rho_{k}-\alpha^{2}-1\right)^{2}\right. \\
& \left.+4\left(\lambda \mu_{2} \rho_{k}-\left(\mu_{1} \rho_{k}+1\right)\left(\mu_{2} \rho_{k}+\alpha^{2}\right)\right)\right]^{1 / 2}
\end{aligned}
$$

It is clear that $\beta_{k}^{-}(\lambda)<\beta_{k}^{+}(\lambda)=0$ if and only if

$$
\begin{aligned}
& \lambda=\frac{1}{\mu_{2} \rho_{k}}\left(\mu_{1} \rho_{k}+1\right)\left(\mu_{2} \rho_{k}+\alpha^{2}\right), \\
& \lambda<\mu_{1} \rho_{k}+\mu_{2} \rho_{k}+\alpha^{2}+1,
\end{aligned}
$$

and $\beta_{k}^{ \pm}(\lambda)= \pm \sigma_{k}(\lambda) i$ with $\sigma_{k} \neq 0$ if and only if

$$
\begin{aligned}
& \lambda=\mu_{1} \rho_{k}+\mu_{2} \rho_{k}+\alpha^{2}+1, \\
& \lambda<\frac{1}{\mu_{2} \rho_{k}}\left(\mu_{1} \rho_{k}+1\right)\left(\mu_{2} \rho_{k}+\alpha^{2}\right) .
\end{aligned}
$$

Thus we introduce two critical numbers

$$
\begin{aligned}
& \lambda_{0}=\min _{\rho_{k}} \frac{1}{\mu_{2} \rho_{k}}\left(\mu_{1} \rho_{k}+1\right)\left(\mu_{2} \rho_{k}+\alpha^{2}\right), \\
& \lambda_{1}=\mu_{1} \rho_{1}+\mu_{2} \rho_{1}+\alpha^{2}+1 .
\end{aligned}
$$

Obviously, the following lemma holds true.

Lemma 3.1. Let $\lambda_{0}$ and $\lambda_{1}$ be the two numbers given by (3.5) and (3.6). Then we have the following assertions:

(1) Let $\lambda_{0}<\lambda_{1}$, and $k_{0} \geq 1$ be the integer such that the minimum is achieved at $\rho_{k_{0}}$ in the definition of $\lambda_{0}$. Then $\beta_{k_{0}}^{+}(\lambda)$ is the first real eigenvalue of (3.1) near $\lambda=\lambda_{0}$ satisfying that

$$
\begin{aligned}
& \beta_{k}^{+}(\lambda)\left\{\begin{array}{ll}
<0 & \text { if } \lambda<\lambda_{0} \\
=0 & \text { if } \lambda=\lambda_{0} \\
>0 & \text { if } \lambda>\lambda_{0}
\end{array} \quad \forall k \in \mathbb{N} \text { with } \rho_{k}=\rho_{k_{0}},\right. \\
& \operatorname{Re} \beta_{j}^{ \pm}\left(\lambda_{0}\right)<0 \quad \forall \beta_{j}^{ \pm} \neq \beta_{k}^{+} \text {with } \rho_{k}=\rho_{k_{0}} .
\end{aligned}
$$

(2) Let $\lambda_{1}<\lambda_{0}$. Then $\beta_{1}^{+}(\lambda)=\bar{\beta}_{1}^{-}(\lambda)$ are a pair of first complex eigenvalues of (3.1) near $\lambda=\lambda_{1}$, and

$$
\begin{aligned}
& \operatorname{Re} \beta_{1}^{+}(\lambda)=\operatorname{Re} \beta_{1}^{-}(\lambda) \begin{cases}<0 & \text { if } \lambda<\lambda_{1}, \\
=0 & \text { if } \lambda=\lambda_{1}, \\
>0 & \text { if } \lambda>\lambda_{1},\end{cases} \\
& \operatorname{Re}_{k}^{ \pm}\left(\lambda_{1}\right)<0 \quad \forall k>1 .
\end{aligned}
$$

Remark 3.1. $\beta_{1}^{ \pm}(\lambda)$ are simple complex eigenvalues at $\lambda_{1}\left(<\lambda_{0}\right)$, and in general, if $\rho_{k_{0}}$ is a simple eigenvalue of (3.2), then $\beta_{k_{0}}^{+}(\lambda)$ is also simple at $\lambda_{0}\left(<\lambda_{1}\right)$. 


\section{TRANSItion From REAL EIGENVALUES}

Hereafter, we always assume that the eigenvalue $\beta_{k_{0}}^{+}$in (3.7) is simple. Based on Lemma 3.1. as $\lambda_{0}<\lambda_{1}$ the transition of (2.7) occurs at $\lambda=\lambda_{0}$, which is from real eigenvalues. Let $\rho_{k_{0}}$ be as in Lemma 3.1, and $e_{k_{0}}$ the eigenvector of (3.2) corresponding to $e_{k_{0}}$ satisfying

$$
\int_{\Omega} e_{k_{0}}^{3} d x \neq 0
$$

Then, under the condition (4.1), for the system (2.3) with (2.4) or with (2.5) we have the following transition theorem.

Theorem 4.1. Let $\lambda_{0}<\lambda_{1}$. Then the system (2.7) has a transition at $\lambda=\lambda_{0}$, which is mixed (Type-III). In particular, the system bifurcates on each side of $\lambda=\lambda_{0}$ to a unique branch $v^{\lambda}$ of steady state solutions, such that the following assertions hold true:

(1) On $\lambda<\lambda_{0}$, the bifurcated solution $v^{\lambda}$ is a saddle, and the stable manifold $\Gamma_{\lambda}^{1}$ of $v^{\lambda}$ separates the space $H$ into two disjoint open sets $U_{1}^{\lambda}$ and $U_{2}^{\lambda}$, such that $v=0 \in U_{1}^{\lambda}$ is an attractor, and the orbits of (2.7) in $U_{2}^{\lambda}$ are far from $v=0$.

(2) On $\lambda>\lambda_{0}$, the stable manifold $\Gamma_{\lambda}^{0}$ of $v=0$ separates the neighborhood $\mathcal{O}$ of $u=0$ into two disjoint open sets $\mathcal{O}_{1}^{\lambda}$ and $\mathcal{O}_{2}^{\lambda}$, such that the transition is jump in $\mathcal{O}_{1}^{\lambda}$, and is continuous in $O_{2}^{\lambda}$. The bifurcated solution $v^{\lambda} \in \mathcal{O}_{2}^{\lambda}$ is an attractor such that for any $\varphi \in \mathcal{O}_{2}^{\lambda}$,

$$
\lim _{t \rightarrow \infty}\left\|v(t, \varphi)-v^{\lambda}\right\|_{H}=0
$$

where $v(t, \varphi)$ is the solution of (2.7) with $v(0, \varphi)=\varphi$.

(3) The bifurcated solution $v^{\lambda}$ can be expressed as

$$
\begin{aligned}
& v^{\lambda}=C \beta_{k_{0}}^{+}(\lambda) \xi_{k_{0}}^{+} e_{k_{0}}+o\left(\left|\beta_{k_{0}}^{ \pm}\right|\right), \\
& \xi_{k_{0}}^{+}=\left(-\mu_{2} \rho_{k_{0}}, \mu_{1} \rho_{k_{0}}+1\right), \\
& C=\frac{\left(\alpha \mu_{2} \rho_{k_{0}}\left(\mu_{2} \rho_{k_{0}}+\alpha^{2}\right)-\alpha^{3}\left(\mu_{1} \rho_{k_{0}}+1\right)\right) \int_{\Omega} e_{k_{0}}^{2} d x}{2 \mu_{2}^{3} \rho_{k_{0}}^{3}\left(\mu_{1} \rho_{k_{0}}+1\right) \int_{\Omega} e_{k_{0}}^{3} d x} .
\end{aligned}
$$

Proof. We apply Theorem A.2 in [8] to prove this theorem. Let $\Phi$ be the center manifold function of (2.7) at $\lambda=\lambda_{0}$. We need to simplify the following expression:

$$
g(y)=\frac{1}{\left(\phi_{k_{0}}^{+}, \phi_{k_{0}}^{+*}\right)}\left(G\left(y \phi_{k_{0}}^{+}+\Phi(y)\right), \phi_{k_{0}}^{+*}\right),
$$

where $y \in \mathbb{R}^{1}, G$ is the operator defined by (2.6), $\phi_{k_{0}}^{+}$is the eigenvector of (2.5) corresponding to $\beta_{k_{0}}^{+}\left(\lambda_{0}\right)=0$, and $\phi_{k_{0}}^{+*}$ is the conjugate eigenvector. By (3.3),

$$
\begin{aligned}
& \phi_{k_{0}}^{+}=\xi_{k_{0}}^{+} e_{k_{0}}, \quad \phi_{k_{0}}^{+*}=\xi_{k_{0}}^{+*} e_{k_{0}}, \\
& \left(\begin{array}{cc}
\lambda_{0}-\left(\mu_{1} \rho_{k_{0}}+1\right) & \alpha^{2} \\
-\lambda_{0} & -\left(\mu_{2} \rho_{k_{0}}+\alpha^{2}\right)
\end{array}\right)\left(\begin{array}{c}
\xi_{k_{0} l}^{+} \\
\xi_{k_{0} 2}^{+}
\end{array}\right)=0, \\
& \left(\begin{array}{cc}
\lambda_{0}-\left(\mu_{1} \rho_{k_{0}}+1\right) & -\lambda_{0} \\
\alpha^{2} & -\left(\mu_{2} \rho_{k_{0}}+\alpha^{2}\right)
\end{array}\right)\left(\begin{array}{c}
\xi_{k_{0} l}^{+*} \\
\xi_{k_{0}}^{+*}
\end{array}\right)=0 .
\end{aligned}
$$


By definition of $\lambda_{0}$ and $k_{0}$, we infer from (4.5) and (4.6) that

$$
\begin{aligned}
& \xi_{k_{0}}^{+}=\left(\xi_{k_{0} 1}^{+}, \xi_{k_{0} 2}^{+}\right)=\left(-\mu_{2} \rho_{k_{0}}, \mu_{1} \rho_{k_{0}}+1\right), \\
& \xi_{k_{0}}^{+*}=\left(\xi_{k_{0} 1}^{+*}, \xi_{k_{0} 2}^{+*}\right)=\left(\mu_{2} \rho_{k_{0}}+\alpha^{2}, \alpha^{2}\right) .
\end{aligned}
$$

Denote by $o(k)=o\left(|y|^{k}\right)$. By $\Phi(y)=o(1)$, the function $g(y)$ in (4.3) is rewritten as

$$
g(y)=\frac{1}{\left(\phi_{k_{0}}^{+}, \phi_{k_{0}}^{+*}\right)}\left(G\left(y \phi_{k_{0}}^{+}\right), \phi_{k_{0}}^{+*}\right)+o(2) .
$$

By (4.4) and (4.7) we see that

$$
G\left(y \phi_{k_{0}}^{+}\right)=\left\{\begin{array}{l}
2 y^{2}\left(\frac{\lambda_{0}}{\alpha} \mu_{2}^{2} \rho_{k}^{2}-\alpha \mu_{2} \rho_{k_{0}}\left(\mu_{1} \rho_{k_{0}}+1\right)\right) e_{+}^{2} k_{0}+o(2), \\
-2 y^{2}\left(\frac{\lambda_{0}}{\alpha} \mu_{2}^{2} \rho_{k}^{2}-\alpha \mu_{2} \rho_{k_{0}}\left(\mu_{1} \rho_{k_{0}}+1\right)\right) e_{k_{0}}^{2}+o(2) .
\end{array}\right.
$$

Thus we deduce from (4.4) and (4.7)-(4.8) that

$$
\begin{gathered}
\left(\phi_{k_{0}}^{+}, \phi_{k_{0}}^{+*}\right)=\left(\alpha^{2}\left(\mu_{1} \rho_{k_{0}}+1\right)-\mu_{2} \rho_{k_{0}}\left(\mu_{2} \rho_{k_{0}}+\alpha^{2}\right) \int_{\Omega} e_{k_{0}}^{2} d x\right. \\
\left(G\left(y \phi_{k_{0}}^{+}, \phi_{k_{0}}^{+*}\right)=y^{2} \cdot \frac{2 \mu_{2}^{3} \rho_{k_{0}}^{3}}{\alpha}\left(\mu_{1} \rho_{k_{0}}+1\right) \int_{\Omega} e_{k_{0}}^{3} d x+o(2) .\right.
\end{gathered}
$$

Therefore the function (4.3) is given by as

$$
g(y)=-\frac{1}{c} y^{2}+o(2)
$$

and the theorem follows from Theorem A.2 in [8. The proof is complete.

Remark 4.1. If the domain $\Omega \neq(0, L) \times D$ with $D \subset \mathbb{R}^{2}$ being a bounded open set, then the condition (4.1) holds true for both the Dirichlet and Neumann boundary conditions (2.4) and (2.5). If $\Omega=(0, L) \times D$, then (4.1) is not true for the Neumann condition (2.5), and is not true for the Dirichlet condition (2.4) if the number $m$ in $\rho_{k_{0}}=\frac{m^{2} \pi^{2}}{L^{2}}+\rho_{k_{0}}^{\prime}$ is even. Here $\rho_{k_{0}}^{\prime}$ is an eigenvalue of the equation on $D$ :

$$
\begin{aligned}
& -\Delta e=\rho_{k_{0}}^{\prime} e \quad \text { for } x \in D, \\
& \left.e\right|_{\partial D}=0 .
\end{aligned}
$$

Now, we consider the case where (4.1) is not true, i.e.,

$$
\int_{\Omega} e_{k_{0}}^{3} d x=0
$$

As mentioned in Remark 4.1, the condition (4.9) may hold true if $\Omega=(0, L) \times D$. We introduce the following parameter

$$
\begin{aligned}
b_{1}= & {\left[\alpha \mu_{2}^{2} \rho_{k_{0}}^{2}\left(\mu_{1} \rho_{k_{0}}+1\right) \int_{\Omega} e_{k_{0}}^{4} d x-2 \alpha^{2} \mu_{2} \rho_{k_{0}} \int_{\Omega} \psi_{2} e_{k_{0}}^{2} d x\right.} \\
& \left.-2\left(\mu_{1} \rho_{k_{0}}+1\right)\left(2 \mu_{2} \rho_{k_{0}}+\alpha^{2}\right) \int_{\Omega} \psi_{1} e_{k_{0}}^{2} d x\right] \\
& \times\left[\alpha^{2}\left(\mu_{1} \rho_{k_{0}}+1\right)-\mu_{2} \rho_{k_{0}}\left(\mu_{2} \rho_{k_{0}}+\alpha^{2}\right)\right]^{-1}
\end{aligned}
$$


where $\psi=\left(\psi_{1}, \psi_{2}\right)$ satisfies

$$
\begin{aligned}
& \mu_{1} \Delta \psi_{1}+\left(\lambda_{0}-1\right) \psi_{1}+\alpha^{2} \psi_{2}=-\frac{2 \mu_{2}^{2} \rho_{k_{0}}^{2}}{\alpha}\left(\mu_{1} \rho_{k_{0}}+1\right) e_{k_{0}}^{2}, \\
& \mu_{2} \Delta \psi_{2}-\lambda_{0} \psi_{1}-\alpha^{2} \psi_{2}=\frac{2 \mu_{2}^{2} \rho_{k_{0}}^{2}}{\alpha}\left(\mu_{1} \rho_{k_{0}}+1\right) e_{k_{0}}^{2}, \\
& \left.\psi\right|_{\partial \Omega}=0 \quad\left(\text { or }\left.\frac{\partial \psi}{\partial n}\right|_{\partial \Omega}=0\right) .
\end{aligned}
$$

By the Fredholm Alternative Theorem, under the condition (4.9), the equation (4.11) has a unique solution.

Theorem 4.2. Let (4.9) hold true, $\lambda_{0}<\lambda_{1}$, and $b_{1}$ is the number given by (4.10). Then the transition of (2.7) at $\lambda=\lambda_{0}$ is continuous if $b_{1}<0$, and is jump if $b_{1}>0$. Moreover, the following assertions hold true:

(1) If $b_{1}>0$, 2.7) has no bifurcation on $\lambda>\lambda_{0}$, and has exact two bifurcated solutions $v_{+}^{\lambda}$ and $v_{-}^{\lambda}$ which are saddles. Moreover, the stable manifolds $\Gamma_{+}^{\lambda}$ and $\Gamma_{-}^{\lambda}$ of the two bifurcated solutions divide $H$ into three disjoint open sets $U_{+}^{\lambda}, U_{0}^{\lambda}, U_{-}^{\lambda}$ such that $v=0 \in U_{0}^{\lambda}$ is an attractor, and the orbits in $U_{ \pm}^{\lambda}$ are far from $v=0$.

(2) If $b_{1}<0$, 2.7) has no bifurcation on $\lambda<\lambda_{0}$, and has exact two bifurcated solutions $v_{+}^{\lambda}$ and $v_{-}^{\lambda}$, which are attractors. In addition, there is a neighborhood $\mathcal{O} \subset H$ of $v=0$, such that the stable manifold $\Gamma$ of $v=0$ divides $\mathcal{O}$ into two disjoint open sets $\mathcal{O}_{+}^{\lambda}$ and $\mathcal{O}_{-}^{\lambda}$ such that $v_{+}^{\lambda} \in \mathcal{O}_{+}^{\lambda}, v_{-}^{\lambda} \in \mathcal{O}_{-}^{\lambda}$, and $v_{ \pm}^{\lambda}$ attracts $\mathcal{O}_{ \pm}^{\lambda}$

(3) The bifurcated solutions $v_{ \pm}^{\lambda}$ can be expressed as

$$
\begin{aligned}
& v_{ \pm}^{\lambda}= \pm C\left(\beta_{k_{0}}^{+}(\lambda)\right)^{1 / 2} \xi_{k_{0}}^{+} e_{k_{0}}+o\left(\left|\beta_{k_{0}}^{+}\right|^{1 / 2}\right), \\
& \xi_{k_{0}}^{+}=\left(-\mu_{2} \rho_{k_{0}}, \mu_{1} \rho_{k_{0}}+1\right), \\
& C=\left[\frac{-\alpha}{\mu_{2} \rho_{k_{0}} b_{1}} \int_{\Omega} e_{k_{0}}^{2} d x\right]^{1 / 2},
\end{aligned}
$$

where $b_{1}$ is as in 4.10 ).

Proof. We use Theorem A.1 in 8 to prove this theorem. To get the function $g(y)$ in (4.3), we need to calculate the center manifold function $\Phi(y)$. By (A.10) in 9 , $\Phi(y)$ satisfies

$$
L_{\lambda_{0}} \Phi=-P_{2} G\left(y \phi_{k_{0}}^{+}\right),
$$

where $P_{2}: H \rightarrow E_{2}$ is the canonical projection, $L_{\lambda}$ is as in (2.6), $\phi_{k_{0}}^{+}$and $\phi_{k_{0}}^{+*}$ are given by (4.4), and

$$
E_{2}=\left\{v \in H \mid\left(v, \phi_{k_{0}}^{+*}\right)=0\right\}
$$

We see that

$$
G\left(y \phi_{k_{0}}^{+}\right)=\left\{\begin{array}{l}
2 \alpha^{-1} \mu_{2}^{2} \rho_{k_{0}}^{2}\left(\mu_{1} \rho_{k_{0}}+1\right) y^{2} e_{k_{0}}^{2}+o(2), \\
-2 \alpha^{-1} \mu_{2}^{2} \rho_{k_{0}}^{2}\left(\mu_{1} \rho_{k_{0}}+1\right) y^{2} e_{k_{0}}^{2}+o(2) .
\end{array}\right.
$$

Let

$$
\Phi=\psi y^{2}+o(2), \quad \psi=\left(\psi_{1}, \psi_{2}\right) \in H
$$


By (4.9), $\left(e_{k_{0}}^{2},-e_{k_{0}}^{2}\right) \in E_{2}$. Hence, it follows from (4.13) and (4.14) that

$$
L_{\lambda_{0}} \psi=-2 \alpha^{-1} \mu_{2}^{2} \rho_{k_{0}}^{2}\left(\mu_{1} \rho_{k_{0}}+1\right)\left(e_{k_{0}}^{2},-e_{k_{0}}^{2}\right),
$$

which is an equivalent form of (4.11).

By (4.14), we have

$$
\begin{aligned}
& G\left(y \phi_{k_{0}}^{+}+\Phi\right)=G\left(y \phi_{k_{0}}^{+}+y^{2} \psi\right)+o(3), \\
& G\left(y \phi_{k_{0}}^{+}+y^{2} \psi\right)=\left\{\begin{array}{r}
O\left(y^{2}\right) e_{k_{0}}^{2}+y^{3}\left[\mu_{2}^{2} \rho_{k_{0}}^{2}\right)\left(\mu_{1} \rho_{k_{0}}+1\right) e_{k_{0}}^{3}-2 \alpha \mu_{2} \rho_{k_{0}} \psi_{2} e_{k_{0}} \\
\left.-2 \alpha^{-1} \psi_{1} e_{k_{0}}\left(\mu_{1} \rho_{k_{0}}+1\right)\left(2 \mu_{2} \rho_{k_{0}}+\alpha^{2}\right)\right]+o(3), \\
O\left(y^{2}\right) e_{k_{0}}^{2}-y^{3}\left[\mu_{2}^{2} \rho_{k_{0}}^{2}\left(\mu_{1} \rho_{k_{0}}+1\right) e_{k_{0}}^{3}-2 \alpha \mu_{2} \rho_{k_{0}} \psi_{2} e_{k_{0}}\right. \\
\left.-2 \alpha^{-1} \psi_{1} e_{k_{0}}\left(\mu_{1} \rho_{k_{0}}+1\right)\left(2 \mu_{2} \rho_{k_{0}}+\alpha^{2}\right)\right]+o(3) .
\end{array}\right.
\end{aligned}
$$

Hence we deduce from (4.4), (4.8) and (4.9) that

$$
\begin{aligned}
\left(G\left(y \phi_{k_{0}}^{+}+\Phi\right), \Phi_{k_{0}}^{+*}\right)= & \frac{\mu_{2} \rho_{k_{0}} y^{3}}{\alpha}\left[\alpha \mu_{2}^{2} \rho_{k_{0}}^{2}\left(\mu_{1} \rho_{k_{0}}+1\right) \int_{\Omega} e_{k_{0}}^{4} d x-2 \alpha^{2} \mu_{2} \rho_{k_{0}} \int_{\Omega} \psi_{2} e_{k_{0}}^{2} d x\right. \\
& \left.-2\left(\mu_{1} \rho_{k_{0}}+1\right)\left(2 \mu_{2} \rho_{k_{0}}+\alpha^{2}\right) \int_{\Omega} \psi_{1} e_{k_{0}}^{2} d x\right]+o(3) .
\end{aligned}
$$

Thus, the function $g(y)$ in (4.3) can be written as

$$
g(y)=\frac{\mu_{2} \rho_{k_{0}} b_{1}}{\alpha \int_{\Omega} e_{k_{0}}^{2} d x} y^{3}+o(3)
$$

where $b_{1}$ is as in (4.10). Hence the theorem follows from Theorem A.1 in 8 .

When the domain $\Omega$ is a rectangle, i.e. $\Omega=\prod_{j=1}^{n}\left(0, L_{j}\right)$, the $b_{1}$ in (4.10) for the Neumann condition can be explicitly expressed in terms of the physical parameters $\mu_{1}, \mu_{2}, \alpha$, and $L_{j}(1 \leq j \leq n)$. For example, we consider the case where $\Omega=(0, L)$. The eigenvalues $\rho_{k}$ and eigenvectors $e_{k}$ of (3.2) are given by

$$
\rho_{k}=\frac{(k-1)^{2} \pi^{2}}{L^{2}}, \quad e_{k}=\cos \frac{(k-1) \pi}{L} x, \quad k=1,2, \cdots .
$$

It is clear that $k_{0} \geq 2$, and (4.9) holds true. We see that

$$
e_{k_{0}}^{2}=\frac{1}{2}\left(1+\cos \frac{2\left(k_{0}-1\right) \pi}{L} x\right)=\frac{1}{2}\left[e_{1}+e_{2 k_{0}-1}\right] .
$$

Hence, by (4.11), we have

$$
\psi=\xi e_{1}+\eta e_{j} \quad \text { with } j=2 k_{0}-1,
$$

where

$$
\begin{aligned}
& \left(\begin{array}{cc}
\lambda_{0}-1 & \alpha^{2} \\
-\lambda_{0} & -\alpha^{2}
\end{array}\right)\left(\begin{array}{l}
\xi_{1} \\
\xi_{2}
\end{array}\right)=\left(\begin{array}{c}
-\frac{1}{\alpha} \mu_{2}^{2} \rho_{k_{0}}^{2}\left(\mu_{1} \rho_{k_{0}}+1\right) \\
\frac{1}{\alpha} \mu_{2}^{2} \rho_{k_{0}}^{2}\left(\mu_{1} \rho_{k_{0}}+1\right)
\end{array}\right), \\
& \left(\begin{array}{cc}
\lambda_{0}-\left(\mu_{1} \rho_{j}+1\right) & \alpha^{2} \\
-\lambda_{0} & -\left(\mu_{2} \rho_{j}+\alpha^{2}\right)
\end{array}\right)\left(\begin{array}{l}
\eta_{1} \\
\eta_{2}
\end{array}\right)=\left(\begin{array}{c}
-\frac{1}{\alpha} \mu_{2}^{2} \rho_{k_{0}}^{2}\left(\mu_{1} \rho_{k_{0}}+1\right) \\
\frac{1}{\alpha} \mu_{2}^{2} \rho_{k_{0}}^{2}\left(\mu_{1} \rho_{k_{0}}+1\right)
\end{array}\right), \\
& \lambda_{0}=\frac{1}{\mu_{2} \rho_{k_{0}}}\left(\mu_{1} \rho_{k_{0}}+1\right)\left(\mu_{2} \rho_{k_{0}}+\alpha^{2}\right) .
\end{aligned}
$$


It is readily to see that

$$
\begin{aligned}
& \xi_{1}=0, \\
& \xi_{2}=-\alpha^{-3} \mu_{2}^{2} \rho_{k_{0}}^{2}\left(\mu_{1} \rho_{k_{0}}+1\right), \\
& \eta_{1}=\frac{\mu_{2}^{3} \rho_{j} \rho_{k_{0}}^{2}\left(\mu_{1} \rho_{k_{0}}+1\right)}{\alpha\left[\left(\mu_{2} \rho_{j}+\alpha^{2}\right)\left(\mu_{1} \rho_{j}+1\right)-\mu_{2} \rho_{j} \lambda_{0}\right]}, \\
& \eta_{2}=\frac{-\mu_{2}^{2} \rho_{k_{0}}^{2}\left(\mu_{1} \rho_{j}+1\right)\left(\mu_{1} \rho_{k_{0}}+1\right)}{\alpha\left[\left(\mu_{2} \rho_{j}+\alpha^{2}\right)\left(\mu_{1} \rho_{j}+1\right)-\mu_{2} \rho_{j} \lambda_{0}\right]} .
\end{aligned}
$$

Inserting (4.16) and (4.17) into (4.10), we derive that

$$
\begin{aligned}
b_{1}= & \frac{\mu_{2}^{2} \rho_{k_{0}}^{2}\left(\mu_{1} \rho_{k_{0}}+1\right) L}{\alpha}\left[\mu_{2} \rho_{k_{0}}+\frac{3}{8} \alpha^{2}\right. \\
& \left.+\frac{\alpha^{2}\left(\mu_{1} \rho_{j}+1\right) \mu_{2} \rho_{k_{0}}-\frac{1}{2}\left(\mu_{1} \rho_{k_{0}}+1\right)\left(2 \mu_{2} \rho_{k_{0}}+\alpha^{2}\right) \mu_{2} \rho_{j}}{\left(\mu_{1} \rho_{j}+1\right)\left(\mu_{2} \rho_{j}+\alpha^{2}\right)-\mu_{2} \rho_{j} \lambda_{0}}\right] \\
& \times\left[\alpha^{2}\left(\mu_{1} \rho_{k_{0}}+1\right)-\mu_{2} \rho_{k_{0}}\left(\mu_{2} \rho_{k_{0}}+\alpha^{2}\right)\right]^{-1}, \\
\rho_{k_{0}}= & \frac{\left(k_{0}-1\right)^{2} \pi^{2}}{L^{2}}, \\
\rho_{j}= & \frac{4\left(k_{0}-1\right)^{2} \pi^{2}}{L^{2}}=4 \rho_{k_{0}} .
\end{aligned}
$$

Thus, for the one dimensional domain $\Omega=(0, L)$, the number $b_{1}$ in (4.18) can be equivalently rewritten as

$$
\begin{aligned}
& b_{1}=\left[\mu_{2} \rho_{k_{0}}+\frac{3}{8} \alpha^{2}-\frac{\mu_{2} \rho_{k_{0}}\left(4 \mu_{1} \mu_{2} \rho_{k_{0}}^{2}+4 \mu_{2} \rho_{k_{0}}-2 \alpha^{2} \mu_{1} \rho_{k_{0}}+\alpha^{2}\right)}{12 \mu_{1} \mu_{2} \rho_{k_{0}}^{2}-3 \alpha^{2}}\right] \\
& \quad \times\left[\alpha^{2}\left(\mu_{1} \rho_{k_{0}}+1\right)-\mu_{2} \rho_{k_{0}}\left(\mu_{2} \rho_{k_{0}}+\alpha^{2}\right)\right]^{-1} .
\end{aligned}
$$

\section{Transition From COMPLEX Eigenvalues}

As $\lambda_{1}<\lambda_{0}$, the transition of (2.7) occurs at $\lambda=\lambda_{1}$, and the system bifurcates to a periodic solution.

We first consider the Neumann boundary condition. In this case, $\lambda_{1}=\alpha^{2}+1$, and we have the following theorem.

Theorem 5.1. For the problem (2.3) with (2.5), when $\lambda_{1}<\lambda_{0}$, the transition at $\lambda=\lambda_{1}$ is continuous, and the problem bifurcates on $\lambda>\lambda_{1}$ to one periodic solution which is an attractor. Moreover, the bifurcated periodic solution $v^{\lambda}=\left(v_{1}^{\lambda}, v_{2}^{\lambda}\right)$ can be expressed as

$$
\begin{aligned}
& v_{1}^{\lambda}=2\left[C \beta_{0}(\lambda)\right]^{1 / 2} \alpha^{2} \sin \left(\alpha t+\frac{\pi}{4}\right)+o\left(\left|\beta_{0}\right|^{1 / 2}\right), \\
& v_{2}^{\lambda}=\left[C \beta_{0}(\lambda)\right]^{1 / 2} \alpha((\alpha-1) \cos \alpha t-(\alpha+1) \sin \alpha t)+o\left(\left|\beta_{0}\right|^{1 / 2}\right),
\end{aligned}
$$

where $\beta_{0}=\frac{1}{2}\left(\lambda-\lambda_{1}\right)$, and $C=\left(2 \pi \alpha^{2}+\frac{3}{2} \pi \alpha^{4}\right)^{-1}$.

Proof. We shall verify this theorem by using Theorem A.3 in 3 . The eigenvalue $\beta_{1}^{ \pm}(\lambda)$ in (3.4) are given by

$$
\beta_{1}^{ \pm}(\lambda)=\frac{1}{2}\left(\lambda-\lambda_{1}\right) \pm \frac{i}{2} \sqrt{4 \alpha^{2}-\left(\lambda-\lambda_{1}\right)^{2}}, \quad\left(\lambda_{1}=\alpha^{2}+1\right) .
$$


Namely, for $\lambda$ near $\lambda_{1}$,

$$
\begin{aligned}
& \beta_{0}(\lambda)=\operatorname{Re} \beta_{1}^{ \pm}(\lambda)=\frac{1}{2}\left(\lambda-\lambda_{1}\right), \\
& \beta_{1}^{ \pm}\left(\lambda_{1}\right)= \pm i \alpha .
\end{aligned}
$$

The eigenvectors $\xi$ and $\eta$ corresponding to $\beta_{1}^{ \pm}\left(\lambda_{1}\right)$ satisfy

$$
\begin{aligned}
& \left(\begin{array}{cc}
\alpha^{2} & \alpha^{2} \\
-\left(\alpha^{2}+1\right) & -\alpha^{2}
\end{array}\right)\left(\begin{array}{l}
\xi_{1} \\
\xi_{2}
\end{array}\right)=\alpha\left(\begin{array}{l}
\eta_{1} \\
\eta_{2}
\end{array}\right), \\
& \left(\begin{array}{cc}
\alpha^{2} & \alpha^{2} \\
-\left(\alpha^{2}+1\right) & -\alpha^{2}
\end{array}\right)\left(\begin{array}{l}
\eta_{1} \\
\eta_{2}
\end{array}\right)=-\alpha\left(\begin{array}{l}
\xi_{1} \\
\xi_{2}
\end{array}\right),
\end{aligned}
$$

It is easy to see that

$$
\begin{aligned}
& \xi=\left(\xi_{1}, \xi_{2}\right)=\left(\alpha^{2}, \alpha(1-\alpha)\right), \\
& \eta=\left(\eta_{1}, \eta_{2}\right)=\left(\alpha^{2},-\alpha(\alpha+1)\right) .
\end{aligned}
$$

The conjugate eigenvectors $\xi^{*}$ and $\eta^{*}$ satisfy

$$
\begin{aligned}
& \left(\begin{array}{cc}
\alpha^{2} & -\left(\alpha^{2}+1\right) \\
\alpha^{2} & -\alpha^{2}
\end{array}\right)\left(\begin{array}{l}
\xi_{1}^{*} \\
\xi_{2}^{*}
\end{array}\right)=\alpha\left(\begin{array}{l}
\eta_{1}^{*} \\
\eta_{2}^{*}
\end{array}\right), \\
& \left(\begin{array}{cc}
\alpha^{2} & -\left(\alpha^{2}+1\right) \\
\alpha^{2} & -\alpha^{2}
\end{array}\right)\left(\begin{array}{l}
\eta_{1}^{*} \\
\eta_{2}^{*}
\end{array}\right)=-\alpha\left(\begin{array}{l}
\xi_{1}^{*} \\
\xi_{2}^{*}
\end{array}\right),
\end{aligned}
$$

leading to

$$
\begin{aligned}
\xi^{*} & =\left(\xi_{1}^{*}, \xi_{2}^{*}\right)=\left(\alpha(\alpha+1), \alpha^{2}\right), \\
\eta^{*} & =\left(e t a_{1}^{*}, \eta_{2}^{*}\right)=\left(\alpha(\alpha-1), \alpha^{2}\right) .
\end{aligned}
$$

It is readily to check that

$$
\begin{aligned}
& \left(\xi, \xi^{*}\right)=-\left(\eta, \eta^{*}\right)=2 \alpha^{3} \int_{\Omega} e_{1}^{2} d x=2 \alpha^{3}|\Omega|, \\
& \left(\xi, \eta^{*}\right)=\left(\eta, \xi^{*}\right)=0 .
\end{aligned}
$$

For the operator $G$ defined by (2.6), we deduce from (5.2) that for $x, y \in \mathbb{R}^{1}$,

$$
\begin{aligned}
G(x \xi+y \eta) & \\
& =\left\{\begin{array}{l}
\frac{2 \lambda_{1}}{\alpha}\left(x \xi_{1}+y \eta_{1}\right)^{2}+2 \alpha\left(x \xi_{1}+y \eta_{1}\right)\left(x \xi_{2}+y \eta_{2}\right)+\left(x \xi_{1}+y \eta_{1}\right)^{2}\left(x \xi_{2}+y \eta_{2}\right), \\
-\frac{2 \lambda_{1}}{\alpha}\left(x \xi_{1}+y \eta_{1}\right)^{2}-2 \alpha\left(x \xi_{1}+y \eta_{1}\right)\left(x \xi_{2}+y \eta_{2}\right)-\left(x \xi_{1}+y \eta_{1}\right)^{2}\left(x \xi_{2}+y \eta_{2}\right),
\end{array}\right. \\
& =\left\{\begin{array}{l}
2 \alpha^{3}\left[(x+y)^{2}+\alpha\left(x^{2}-y^{2}\right)\right]+\alpha^{5}\left[(x+y)\left(x^{2}-y^{2}\right)-\alpha(x+y)^{3}\right], \\
-2 \alpha^{3}\left[(x+y)^{2}+\alpha\left(x^{2}-y^{2}\right)\right]-\alpha^{5}\left[(x+y)\left(x^{2}-y^{2}\right)-\alpha(x+y)^{3}\right] .
\end{array}\right.
\end{aligned}
$$

Because the first eigenvector space $E_{1}=\operatorname{span}\{\xi, \eta\}$ of (3.1) with (2.5) is invariant for the equations (2.3) with (2.5), the center manifold function $\Phi$ vanishes, i.e.,

$$
\Phi(x, y) \equiv 0 .
$$


Therefore, we derive from (5.2)-(5.5) that

$$
\begin{aligned}
& \frac{\left(G(x \xi+y \eta+\Phi), \xi^{*}\right)}{\left(\xi, \xi^{*}\right)}=a_{20} x^{2}+a_{11} x y+a_{02} y^{2}+a_{30} x^{3}+a_{21} x^{2} y+a_{12} x y^{2}+a_{03} y^{3}, \\
& \frac{\left(G(x \xi+y \eta+\Phi), \eta^{*}\right)}{\left(\eta, \eta^{*}\right)}=b_{20} x^{2}+b_{11} x y+b_{02} y^{2}+b_{30} x^{3}+b_{21} x^{2} y+b_{12} x y^{2}+b_{03} y^{3},
\end{aligned}
$$

where

$$
\begin{aligned}
a_{20} & =\alpha(\alpha+1), & a_{02} & =\alpha(1-\alpha), \\
b_{20} & =\alpha(\alpha+1), & b_{02} & =\alpha(1-\alpha), \\
a_{11} & =2 \alpha, & b_{11} & =2 \alpha, \\
a_{30} & =\frac{1}{2} \alpha^{3}(1-\alpha), & b_{03} & =-\frac{1}{2} \alpha^{3}(1+\alpha), \\
a_{12} & =-\frac{1}{2} \alpha^{3}(1+3 \alpha), & b_{21} & =\frac{1}{2} \alpha^{3}(1-3 \alpha) .
\end{aligned}
$$

Then, the parameter $b$ in Theorem A.3 in $[3]$ is

$$
\begin{aligned}
b= & \frac{\pi}{2 \alpha}\left(a_{02} b_{02}-a_{20} b_{20}\right)+\frac{\pi}{4 \alpha}\left(a_{11} a_{20}+a_{11} a_{02}-b_{11} b_{20}-b_{11} b_{02}\right) \\
& +\frac{3 \pi}{4}\left(a_{30}+b_{03}\right)+\frac{\pi}{4}\left(a_{12}+b_{21}\right) \\
= & -\pi \alpha^{2}\left(2+\frac{3}{2} \alpha^{2}\right) .
\end{aligned}
$$

Namely, $b<0$. Hence, by Theorem A.3 in 3, the system (2.7) bifurcates from $(v, \lambda)=\left(0, \lambda_{1}\right)$ to a periodic solution on $\lambda>\lambda_{1}$, which is an attractor. The proof of the expression (5.1) is classical. Thus the theorem is proved.

Now, we consider the Dirichlet boundary condition. In this case, $\rho_{1}>0$ and $\lambda_{1}=\left(\mu_{1}+\mu_{2}\right) \rho_{1}+\alpha^{2}+1$. By (3.5) and (3.6) it is easy to see that as $\lambda_{1}<\lambda_{0}$ we have

$$
\mu_{2} \rho_{1}\left(\mu_{2} \rho_{1}+\alpha^{2}\right)<\alpha^{2}\left(\mu_{1} \rho_{1}+1\right) .
$$

Then we define the following parameter

$$
\begin{aligned}
b_{1}= & \frac{2 \pi \alpha^{2}\left[\int_{\Omega} e_{1}^{3} d x\right]^{2}}{\sigma_{0}^{2}\left[\int_{\Omega} e_{1}^{2} d x\right]^{2}}\left(\mu_{1} \rho_{1}+1\right)\left(\mu_{2}^{2} \rho_{1}^{2}+2 \mu_{2} \rho_{1}\left(\mu_{1} \rho_{1}+1\right)-\sigma_{0}^{2}\right) \\
& -\frac{\pi \alpha^{2} \int_{\Omega} e_{1}^{4} d x}{2 \int_{\Omega} e_{1}^{2} d x}\left(2 \mu_{2} \rho_{1}+3 \alpha^{2}\right) \\
& +\frac{2 \pi \alpha^{2}}{\int_{\Omega} e_{1}^{2} d x}\left[\left(3 \mu_{1} \rho_{1}+\mu_{2} \rho_{1}+3\right) A_{1}+\left(\mu_{1} \mu_{2} \rho_{1}^{2}+\mu_{2} \rho_{1}+\delta_{0}\right) B_{1}\right] \\
& -\frac{8 \pi \alpha^{2} \sigma_{0}^{2}}{\int_{\Omega} e_{1}^{2} d x}\left[\left(\mu_{1} \rho_{1}+\mu_{2} \rho_{1}+1\right) A_{2}-\left(\mu_{1} \mu_{2} \rho_{1}^{2}+\mu_{2} \rho_{1}-\sigma_{0}\right) B_{2}\right] \\
& -\frac{4 \pi \alpha^{2}}{\int_{\Omega} e_{1}^{2} d x}\left[\left(\mu_{1} \mu_{2} \rho_{1}^{2}+\mu_{2} \rho_{1}-\sigma_{0}^{2}\right) A_{3}+\sigma_{0}^{2}\left(\mu_{1} \rho_{1}+\mu_{2} \rho_{1}+1\right) B_{3}\right],
\end{aligned}
$$


where

$$
\begin{aligned}
\sigma_{0} & =\left[\alpha^{2}\left(\mu_{1} \rho_{1}+1\right)-\mu_{2} \rho_{1}\left(\mu_{2} \rho_{1}+\alpha^{2}\right)\right]^{1 / 2}, \\
A_{i} & =\left(2 \lambda_{1}-\mu_{2} \rho_{1}-\alpha^{2}\right) B_{i}+\alpha^{2} C_{i} \quad \text { for } i=1,2,3, \\
B_{1} & =\sum_{k>1}^{\infty} \frac{\mu_{2} \rho_{k}\left[\int_{\Omega} e_{1}^{2} e_{k} d x\right]^{2}}{\operatorname{det} M_{k} \int_{\Omega} e_{k}^{2} d x} \\
B_{2}= & \sum_{k>1}^{\infty} \frac{\left[\int_{\Omega} e_{1}^{2} e_{k} d x\right]^{2}}{\operatorname{det} M_{k} \operatorname{det}\left(M_{k}^{2}+4 \sigma_{0}^{2}\right) \int_{\Omega} e_{k}^{2} d x}\left[\left(\mu_{2} \rho_{k}+\alpha^{2}\right) D_{k}+\alpha^{2} Q_{k}\right], \\
B_{3}= & \sum_{k>1}^{\infty} \frac{\left[\int_{\Omega} e_{1}^{2} e_{k} d x\right]^{2}}{\operatorname{det}\left(M_{k}^{2}+4 \sigma_{0}^{2}\right) \int_{\Omega} e_{k}^{2} d x} D_{k}, \\
C_{1}= & -\sum_{k>1}^{\infty} \frac{\left(\mu_{1} \rho_{1}+1\right)\left[\int_{\Omega} e_{1}^{2} e_{k} d x\right]^{2}}{\operatorname{det} M_{k} \int_{\Omega} e_{k}^{2} d x}, \\
C_{2}= & \sum_{k>1}^{\infty} \frac{\left[\int_{\Omega} e_{1}^{2} e_{k} d x\right]^{2}}{\operatorname{det} M_{k} \operatorname{det}\left(M_{k}^{2}+4 \sigma_{0}^{2}\right) \int_{\Omega} e_{k}^{2} d x}\left[\left(\mu_{1} \rho_{k}+1-\lambda_{1}\right) Q_{k}-\lambda_{1} D_{k}\right], \\
C_{3}= & \sum_{k>1}^{\infty} \frac{\left[\int_{\Omega} e_{1}^{2} e_{k} d x\right]^{2}}{\operatorname{det}\left(M_{k}^{2}+4 \sigma_{0}^{2}\right) \int_{\Omega} e_{k}^{2} d x} Q_{k}, \\
D_{k}= & \left(\mu_{2} \rho_{k}+\alpha^{2}\right)^{2}+4 \sigma_{0}^{2}-\alpha^{2}\left(\mu_{1}+\mu_{2}\right) \rho_{k}-\alpha^{2}\left(\alpha^{2}+1\right), \\
Q_{k}= & \lambda_{1} \alpha^{2}-\lambda_{1}\left(\mu_{1}+\mu_{2}\right)\left(\rho_{k}-\rho_{1}\right)-\left(\mu_{1} \rho_{k}+1-\lambda_{1}\right)^{2}-4 \sigma_{0}^{2} .
\end{aligned}
$$

Here $M_{k}$ is the matrix defined by

$$
M_{k}=\left(\begin{array}{cc}
-\mu_{1} \rho_{k}+\lambda_{1}-1 & \alpha^{2} \\
-\lambda_{1} & -\mu_{2} \rho_{k}-\alpha^{2}
\end{array}\right) .
$$

Theorem 5.2. Let $b_{1}$ be the number given by (5.6) and $\lambda_{1}<\lambda_{0}$. For the problem (2.3)- 2.4), the following assertions hold true:

(1) The problem undergoes a dynamic transition at $\lambda=\lambda_{1}$, which is the Hopf bifurcation.

(2) When $b_{1}<0$, the transition is of Type-I and bifurcates to a stable periodic solution on $\lambda>\lambda_{1}$, and when $b_{1}>0$ the transition is of Type-II and bifurcates to an unstable periodic solution on $\lambda<\lambda_{1}$.

(3) The bifurcated periodic solution $v^{\lambda}=\left(v_{1}^{\lambda}, v_{2}^{\lambda}\right)$ can be expressed as

$$
\begin{aligned}
& v_{1}^{\lambda}=2 \alpha^{2}\left[-\gamma(\lambda) / b_{1}\right]^{1 / 2} e_{1} \sin \left(\sigma_{0} t+\frac{\pi}{4}\right)+o\left(|\gamma|^{1 / 2}\right), \\
& v_{2}^{\lambda}=2\left(\sigma_{0}^{2}+\left(\mu_{2} \rho_{1}+\alpha^{2}\right)^{2}\right)\left[-\frac{\gamma(\lambda)}{b_{1}}\right]^{12} e_{1} \cos \left(\sigma_{0} t+\theta\right)+o\left(|\gamma|^{1 / 2}\right) \text {, } \\
& \theta=\tan ^{-1} \frac{\sigma_{0}+\mu_{2} \rho_{1}+\alpha^{2}}{\sigma_{0}-\mu_{2} \rho_{1}-\alpha^{2}},
\end{aligned}
$$

Proof. By (3.3) the eigenvalues and eigenvectors of (3.1) with (2.4) at $\lambda_{1}=\left(\mu_{1}+\right.$ $\left.\mu_{2}\right) \rho_{1}+\alpha^{2}+1$ are determined by the matrices $M_{k}$ given by (5.7). It is clear that $M_{1}$ has a pair of imaginary eigenvalues

$$
\beta_{1}^{ \pm}\left(\lambda_{1}\right)= \pm i \sigma_{0} \quad\left(\sigma_{0} \text { as in (15.6) }\right) .
$$


Let $\tilde{\xi}, \tilde{\eta} \in \mathbb{R}^{2}$ be the eigenvectors of $M_{1}$ satisfying

$$
M_{1} \tilde{\xi}=\sigma_{0} \tilde{\eta}, \quad M_{1} \tilde{\eta}=-\sigma_{0} \tilde{\xi}
$$

Then, by (3.3) the eigenvectors of (3.1) corresponding to $\beta_{1}^{ \pm}\left(\lambda_{1}\right)$ are given by $\xi=$ $\tilde{\xi} e_{1}$ and $\eta=\tilde{\eta} e_{1}$. It is readily to check that

$$
\begin{aligned}
& \xi=\left(\xi_{1}, \xi_{2}\right)=\left(\alpha^{2} e_{1},\left(\sigma_{0}-\mu_{2} \rho_{1}-\alpha^{2}\right) e_{1}\right), \\
& \eta=\left(\eta_{1}, \eta_{2}\right)=\left(\alpha^{2} e_{1},-\left(\sigma_{0}+\mu_{2} \rho_{1}+\alpha^{2}\right) e_{1}\right) .
\end{aligned}
$$

We consider the conjugate eigenvectors $\xi^{*}=\tilde{\xi}^{*} e_{1}$ and $\eta^{*}=\tilde{\eta}^{*} e_{1}$ with

$$
M_{1}^{*} \tilde{\xi}^{*}=\sigma_{0} \tilde{\eta}^{*}, \quad M_{1}^{*} \tilde{\eta}^{*}=-\sigma_{0} \tilde{\xi}^{*}
$$

where $M_{1}^{*}$ is the transpose of $M_{1}$. Direct calculation shows that

$$
\begin{aligned}
& \xi^{*}=\left(\xi_{1}^{*}, \xi_{2}^{*}\right)=\left(\left(\sigma_{0}+\mu_{2} \rho_{1}+\alpha^{2}\right) e_{1}, \alpha^{2} e_{1}\right), \\
& \eta^{*}=\left(\eta_{1}^{*}, \eta_{2}^{*}\right)=\left(\left(-\sigma_{0}+\mu_{2} \rho_{1}+\alpha^{2}\right) e_{1}, \alpha^{2} e_{1}\right) .
\end{aligned}
$$

It is easy to see that

$$
\begin{aligned}
& \left(\xi, \eta^{*}\right)=\left(\eta, \xi^{*}\right)=0, \\
& \left(\xi, \xi^{*}\right)=-\left(\eta, \eta^{*}\right)=2 \alpha^{2} \sigma_{0} \int_{\Omega} e_{1}^{2} d x .
\end{aligned}
$$

Let $u=x \xi+y \eta+\Phi(x, y) \in H$ be a solution of (2.3)-(2.4) at $\lambda=\lambda_{1}$, and $\Phi$ be the center manifold function. By (5.13) the reduced equations of (2.3)-(2.4) read

$$
\begin{aligned}
& \frac{d x}{d t}=-\sigma_{0} y+\frac{1}{\left(\xi, \xi^{*}\right)}\left(G(x \xi+y \eta+\Phi), \xi^{*}\right), \\
& \frac{d y}{d t}=\sigma_{0} x+\frac{1}{\left(\eta, \eta^{*}\right)}\left(G(x \xi+y \eta+\Phi), \eta^{*}\right),
\end{aligned}
$$

where the operator $G$ is given by

$$
G(u)=G_{2}(u)+G_{3}(u),
$$

and $G_{k}(k=2,3)$ is a $k$-multilinear operator defined by

$$
\begin{aligned}
& G_{2}(u, v)=2\left(\frac{\lambda_{1}}{\alpha} u_{1} v_{1}+\alpha u_{1} v_{2},-\left(\frac{\lambda_{1}}{\alpha} u_{1} v_{1}+\alpha u_{1} v_{2}\right)\right), \\
& G_{3}(u, v, w)=\left(u_{1} v_{1} w_{2},-u_{1} v_{1} w_{2}\right), \\
& G_{2}(u)=G_{2}(u, u), \\
& G_{3}(u)=G_{3}(u, u, u) .
\end{aligned}
$$


Based on (5.9)-(5.13) and (5.15)-(5.16), (5.14) are rewritten as

$$
\begin{aligned}
\frac{d x}{d t}= & -\sigma_{0} y+a_{20} x^{2}+a_{11} x y+a_{02} y^{2}+a_{30} x^{3}+a_{21} x^{2} y \\
& +a_{12} x y^{2}+a_{03} y^{3}+\frac{x}{\left(\xi, \xi^{*}\right)}\left(G_{2}(\xi, \Phi)+G_{2}(\Phi, \xi), \xi^{*}\right) \\
& +\frac{y}{\left(\xi, \xi^{*}\right)}\left(G_{2}(\eta, \Phi)+G(\Phi, \eta), \eta^{*}\right)+o(3), \\
\frac{d y}{d t}= & \sigma_{0} x+b_{20} x^{2}+b_{11} x y+b_{22} y^{2}+b_{30} x^{3}+b_{21} x^{2} y \\
& +b_{12} x y^{2}+b_{03} y^{3}+\frac{x}{\left(\eta, \eta^{*}\right)}\left(G_{2}(\xi, \Phi)+G_{2}(\Phi, \xi), \eta^{*}\right) \\
& +\frac{y}{\left(\eta, \eta^{*}\right)}\left(G_{2}(\eta, \Phi)+G_{2}(\Phi, \eta), \eta^{*}\right)+o(3),
\end{aligned}
$$

where

$$
\begin{aligned}
a_{20}= & \frac{\left(G_{2}(\xi, \xi), \xi^{*}\right)}{\left(\xi, \xi^{*}\right)}=\frac{\alpha \int_{\Omega} e_{1}^{3} d x}{\sigma_{0} \int_{\Omega} e_{1}^{2} d x}\left(\sigma_{0}+\mu_{2} \rho_{1}\right)\left(\mu_{1} \rho_{1}+1+\sigma_{0}\right), \\
a_{11}= & \frac{\left(G_{2}(\xi, \eta)+G_{2}(\eta, \xi), \xi^{*}\right)}{\left(\xi, \xi^{*}\right)}=\frac{2 \alpha \int_{\Omega} e_{1}^{3} d x}{\sigma_{0} \int_{\Omega} e_{1}^{2} d x}\left(\sigma_{0}+\mu_{2} \rho_{1}\right)\left(\mu_{1} \rho_{1}+1\right), \\
a_{02} & =\frac{\left(G_{2}(\eta, \eta), \xi^{*}\right)}{\left(\xi, \xi^{*}\right)}=\frac{\alpha \int_{\Omega} e_{1}^{3} d x}{\sigma_{0} \int_{\Omega} e_{1}^{2} d x}\left(\sigma_{0}+\mu_{2} \rho_{1}\right)\left(\mu_{1} \rho_{1}+1-\sigma_{0}\right), \\
a_{30}= & \frac{\left(G_{3}(\xi, \xi, \xi), \xi^{*}\right)}{\left(\xi, \xi^{*}\right)}=\frac{\alpha^{2} \int_{\Omega} e_{1}^{4} d x}{2 \sigma_{0} \int_{\Omega} e_{1}^{2} d x}\left(\sigma_{0}+\mu_{2} \rho_{1}\right)\left(\sigma_{0}-\mu_{2} \rho_{1}-\alpha^{2}\right), \\
a_{21}= & \frac{1}{\left(\xi, \xi^{*}\right)}\left(G_{3}(\xi, \xi, \eta)+G_{3}(\xi, \eta, \xi)+\left(G_{3}(\eta, \xi, \xi), \xi^{*}\right)\right. \\
= & \frac{\alpha^{2} \int_{\Omega} e_{1}^{4} d x}{2 \sigma_{0} \int_{\Omega} e_{1}^{2} d x}\left(\sigma_{0}+\mu_{2} \rho_{1}\right)\left(\sigma_{0}-3 \mu_{2} \rho_{1}-3 \alpha^{2}\right), \\
a_{12}= & \frac{1}{\left(\xi, \xi^{*}\right)}\left(G_{3}(\xi, \eta, \eta)+G_{3}(\eta, \xi, \eta)+G_{3}(\eta, \eta, \xi), \xi^{*}\right) \\
= & -\frac{\alpha^{2} \int_{\Omega} e_{1}^{4} d x}{2 \sigma_{0} \int_{\Omega} e_{1}^{2} d x}\left(\sigma_{0}+\mu_{2} \rho_{1}\right)\left(\sigma_{0}+3 \mu_{2} \rho_{1}+3 \alpha^{2}\right), \\
a_{03}= & \frac{\left(G_{3}(\eta, \eta, \eta), \xi^{*}\right)}{\left(\xi, \xi^{*}\right)}=-\frac{\alpha^{2} \int_{\Omega} e_{1}^{4} d x}{2 \sigma_{0} \int_{\Omega} e_{1}^{2} d x}\left(\sigma_{0}+\mu_{2} \rho_{1}\right)\left(\sigma_{0}+\mu_{2} \rho_{1}+\alpha^{2}\right),
\end{aligned}
$$


and

$$
\begin{aligned}
b_{20} & =\frac{\left(G_{2}(\xi, \xi), \eta^{*}\right)}{\left(\eta, \eta^{*}\right)}=\frac{\alpha \int_{\Omega} e_{1}^{3} d x}{\sigma_{0} \int_{\Omega} e_{1}^{2} d x}\left(\sigma_{0}-\mu_{2} \rho_{1}\right)\left(\mu_{1} \rho_{1}+1+\sigma_{0}\right), \\
b_{11} & =\frac{\left(G_{2}(\xi, \eta)+G(\eta, \xi), \eta^{*}\right)}{\left(\eta, \eta^{*}\right)}=\frac{2 \alpha \int_{\Omega} e_{1}^{3} d x}{\sigma_{0} \int_{\Omega} e_{1}^{2} d x}\left(\sigma_{0}-\mu_{2} \rho_{1}\right)\left(\mu_{1} \rho_{1}+1\right), \\
b_{02} & =\frac{\left(G_{2}(\eta, \eta), \eta^{*}\right)}{\left(\eta, \eta^{*}\right)}=\frac{\alpha \int_{\Omega} e_{1}^{3} d x}{\sigma_{0} \int_{\Omega} e_{1}^{2} d x}\left(\sigma_{0}-\mu_{2} \rho_{1}\right)\left(\mu_{1} \rho_{1}+1-\sigma_{0}\right), \\
b_{30} & =\frac{\left(G_{3}(\xi, \xi, \xi), \eta^{*}\right)}{\left(\eta, \eta^{*}\right)}=\frac{\alpha^{2} \int_{\Omega} e_{1}^{4} d x}{2 \sigma_{0} \int_{\Omega} e_{1}^{2} d x}\left(\sigma_{0}-\mu_{2} \rho_{1}\right)\left(\sigma_{0}-\mu_{2} \rho_{1}-\alpha^{2}\right), \\
b_{21} & =\frac{1}{\left(\eta, \eta^{*}\right)}\left(G_{3}(\xi, \xi, \eta)+G_{3}(\xi, \eta, \xi)+G(\eta, \xi, \xi), \eta^{*}\right) \\
& =\frac{\alpha^{2} \int_{\Omega} e_{1}^{4} d x}{2 \sigma_{0} \int_{\Omega} e_{1}^{2} d x}\left(\sigma_{0}-\mu_{2} \rho_{1}\right)\left(\sigma_{0}-3 \mu_{2} \rho_{1}-3 \alpha^{2}\right), \\
b_{12}= & \frac{1}{\left(\eta, \eta^{*}\right)}\left(G_{3}(\eta, \eta, \xi)+G_{3}(\eta, \xi, \eta)+G_{3}(\xi, \eta, \eta), \eta^{*}\right) \\
= & -\frac{\alpha^{2} \int_{\Omega} e_{1}^{4} d x}{2 \sigma_{0} \int_{\Omega} e_{1}^{2} d x}\left(\sigma_{0}-\mu_{2} \rho_{1}\right)\left(\sigma_{0}+3 \mu_{2} \rho_{1}+3 \alpha^{2}\right), \\
b_{03}= & \frac{\left(G_{3}(\eta, \eta, \eta), \eta^{*}\right)}{\left(\eta, \eta^{*}\right)}=-\frac{\alpha^{2} \int_{\Omega} e_{1}^{4} d x}{2 \sigma_{0} \int_{\Omega} e_{1}^{2} d x}\left(\sigma_{0}-\mu_{2} \rho_{1}\right)\left(\sigma_{0}+\mu_{2} \rho_{1}+\alpha^{2}\right) .
\end{aligned}
$$

We are now in a position to derive the center manifold function $\Phi$. By (A.10) in 9],

$$
\Phi=\Phi_{1}+\Phi_{2}+\Phi_{3}+o(2)
$$

where

$$
\begin{aligned}
& -L_{\lambda_{1}} \Phi_{1}=P_{2}\left[G_{2}(\xi, \xi) x^{2}+\left(G_{2}(\xi, \eta)+G_{2}(\eta, \xi)\right) x y+G_{2}(\eta, \eta) y^{2}\right] \\
& -\left[L_{\lambda_{1}}^{2}+4 \sigma_{0}^{2}\right] L_{\lambda_{1}} \Phi_{2}=2 \sigma_{0}^{2} P_{2}\left[\left(G_{2}(\xi, \xi)-G_{2}(\eta, \eta)\right)\left(y^{2}-x^{2}\right)-2\left(G_{2}(\xi, \eta)+G_{2}(\eta, \xi)\right) x y\right] \\
& {\left[L_{\lambda_{1}}^{2}+4 \sigma_{0}^{2}\right] \Phi_{3}=\sigma_{0} P_{2}\left[\left(G_{2}(\xi, \eta)+G_{2}(\eta, \xi)\right)\left(y^{2}-x^{2}\right)+2\left(G_{2}(\xi, \xi)-G_{2}(\eta, \eta)\right) x y\right]}
\end{aligned}
$$

$L_{\lambda_{1}}=A+B_{\lambda_{1}}$ is the linear operator defined by (2.6), $P_{2}: H \rightarrow E_{2}$ the canonical projection, and $E_{2}=\left\{u \in H \mid\left(u, \xi^{*}\right)=0,\left(u, \eta^{*}\right)=0\right\}$ is the complement of $E_{1}=$ $\operatorname{span}\{\xi, \eta\}$ in $H$. Note that the eigenvectors of $L_{\lambda_{1}}$ satisfy

$$
\begin{aligned}
& \phi_{k}=\tilde{\phi}_{k} e_{1}, \quad \tilde{\phi}_{k} \in \mathbb{R}^{2}, \quad k=1,2, \cdots, \\
& M_{k} \tilde{\phi}_{k}=\beta_{k} \tilde{\phi}_{k} \quad\left(M_{k} \text { the matrix as in (5.7) }\right) .
\end{aligned}
$$


Hence, we obtain from (5.9), (5.10), (5.16) and (5.18) that

$$
\begin{aligned}
\Phi_{1}= & 2 \alpha^{3}\left[\left(\mu_{1} \rho_{1}+\sigma_{0}+1\right) x^{2}+2\left(\mu_{1} \rho_{1}+1\right) x y+\left(\mu_{1} \rho_{1}-\sigma_{0}+1\right) y^{2}\right] \\
& \times \sum_{k>1}^{\infty} \frac{\int_{\Omega} e_{1}^{2} e_{k} d x}{\int_{\Omega} e_{k}^{2} d x}\left(-M_{k}\right)^{-1}\left(\begin{array}{c}
1 \\
-1
\end{array}\right) e_{k}, \\
\Phi_{2}= & 8 \alpha^{3} \sigma_{0}^{2}\left[\sigma_{0}\left(y^{2}-x^{2}\right)-2\left(\mu_{1} \rho_{1}+1\right) x y\right] \sum_{k>1}^{\infty} \frac{\int_{\Omega} e_{1}^{2} e_{k} d x}{\int_{\Omega} e_{k}^{2} d x}\left(-M_{k}\right)^{-1}\left(M_{k}^{2}+4 \sigma_{0}^{2}\right)^{-1}\left(\begin{array}{c}
1 \\
-1
\end{array}\right) e_{k}, \\
\Phi_{3}= & 4 \alpha^{3} \sigma_{0}\left[\left(\mu_{1} \rho_{1}+1\right)\left(y^{2}-x^{2}\right)+2 \sigma_{0} x y\right] \sum_{k>1}^{\infty} \frac{\int_{\Omega} e_{1}^{2} e_{k} d x}{\int_{\Omega} e_{k}^{2} d x}\left(M_{k}^{2}+4 \sigma_{0}^{2}\right)^{-1}\left(\begin{array}{c}
1 \\
-1
\end{array}\right) e_{k} .
\end{aligned}
$$

Direct calculation shows that

$$
\begin{aligned}
& \left(-M_{k}\right)^{-1}=\frac{1}{\operatorname{det} M_{k}}\left[\begin{array}{cc}
\mu_{2} \rho_{k}+\alpha^{2} & \alpha^{2} \\
-\lambda_{1} & \mu_{1} \rho_{k}+1-\lambda_{1}
\end{array}\right], \\
& \left(M_{k}^{2}+4 \sigma_{0}^{2}\right)^{-1}=\frac{1}{\operatorname{det}\left(M_{k}^{2}+4 \sigma_{0}^{2}\right)}\left[\begin{array}{cc}
\left(\mu_{2} \rho_{k}+\alpha^{2}\right)^{2}+4 \sigma_{0}^{2}-\lambda_{1} \alpha^{2} & \alpha^{2}\left(\mu_{1}+\mu_{2}\right)\left(\rho_{k}-\rho_{1}\right) \\
-\lambda_{1}\left(\mu_{1}+\mu_{2}\right)\left(\rho_{k}-\rho_{1}\right) & \left(\mu_{1} \rho_{k}+1-\lambda_{1}\right)^{2}+4 \sigma_{0}^{2}-\lambda_{1} \alpha^{2}
\end{array}\right] .
\end{aligned}
$$

Thus we have

$$
\begin{aligned}
& \Phi_{1}=2 \alpha^{3}\left[\left(\mu_{1} \rho_{1}+1+\sigma_{0}\right) x^{2}+2\left(\mu_{1} \rho_{1}+1\right) x y+\left(\mu_{1} \rho_{1}+1-\sigma_{0}\right) y^{2}\right]\left(\begin{array}{c}
E_{1} \\
F_{1}
\end{array}\right), \\
& \Phi_{2}=8 \alpha^{3} \sigma_{0}^{2}\left[-\sigma_{0} x^{2}-2\left(\mu_{1} \rho_{1}+1\right) x y+\sigma_{0} y^{2}\right]\left(\begin{array}{c}
E_{2} \\
F_{2}
\end{array}\right), \\
& \Phi_{3}=4 \alpha^{3} \sigma_{0}\left[-\left(\mu_{1} \rho_{1}+1\right) x^{2}+2 \sigma_{0} x y+\left(\mu_{1} \rho_{1}+1\right) y^{2}\right]\left(\begin{array}{c}
E_{3} \\
F_{3}
\end{array}\right),
\end{aligned}
$$

where

$$
\begin{aligned}
& E_{1}=\sum_{k>1}^{\infty} \frac{\int_{\Omega} e_{1}^{2} e_{k} d x}{\operatorname{det} M_{k} \int_{\Omega} e_{k}^{2} d x} \mu_{2} \rho_{k} e_{k}, \\
& F_{1}=-\sum_{k>1}^{\infty} \frac{\int_{\Omega} e_{1}^{2} e_{k} d x}{\operatorname{det} M_{k} \int_{\Omega} e_{k}^{2} d x}\left(\mu_{1} \rho_{k}+1\right) e_{k}, \\
& E_{2}=\sum_{k>1}^{\infty} \frac{\int_{\Omega} e_{1}^{2} e_{k} d x}{\operatorname{det} M_{k} \operatorname{det}\left(M_{k}^{2}+4 \sigma_{0}^{2}\right) \int_{\Omega} e_{k}^{2} d x}\left(\left(\mu_{2} \rho_{k}+\alpha^{2}\right) D_{k}+\alpha^{2} Q_{k}\right) e_{k}, \\
& F_{2}=\sum_{k>1}^{\infty} \frac{\int_{\Omega} e_{1}^{2} e_{k} d x}{\operatorname{det} M_{k} \operatorname{det}\left(M_{k}^{2}+4 \sigma_{0}^{2}\right) \int_{\Omega} e_{k}^{2} d x}\left(\left(\mu_{1} \rho_{k}+1-\lambda_{1}\right) Q_{k}-\lambda_{1} D_{k}\right) e_{k}, \\
& E_{3}=\sum_{k>1}^{\infty} \frac{\int_{\Omega} e_{1}^{2} e_{k} d x}{\operatorname{det}\left(M_{k}^{2}+4 \sigma_{0}^{2}\right) \int_{\Omega} e_{k}^{2} d x} D_{k} e_{k}, \\
& F_{3}=\sum_{k>1}^{\infty} \frac{\int_{\Omega} e_{1}^{2} e_{k} d x}{\operatorname{det}\left(M_{k}^{2}+4 \sigma_{0}^{2}\right) \int_{\Omega} e_{k}^{2} d x} Q_{k} e_{k} .
\end{aligned}
$$


Inserting $\Phi=\Phi_{1}+\Phi_{2}+\Phi_{3}+o(2)$ into (5.17) we derive that

$$
\begin{aligned}
& \frac{d x}{d t}=-\sigma_{0} y+\sum_{2 \leq i+j \leq 3} a_{i j} x^{i} y^{j}+\sum_{k+r=3} \tilde{a}_{k r} x^{k} y^{r}+o(3), \\
& \frac{d y}{d t}=\sigma_{0} x+\sum_{2 \leq i+j \leq 3} b_{i j} x^{i} y^{j}+\sum_{k+r=3} \tilde{b}_{k r} x^{k} y^{r}+o(3),
\end{aligned}
$$

where $a_{i j}$ and $b_{i j}(0 \leq i, j \leq 3)$ are as in (5.17), and

$$
\begin{aligned}
\tilde{a}_{30}= & \frac{2 \alpha^{2}\left(\sigma_{0}+\mu_{2} \rho_{1}\right)}{\sigma_{0} \int_{\Omega} e_{1}^{2} d x}\left[\left(\mu_{1} \rho_{1}+1+\sigma_{0}\right) I_{1}-4 \sigma_{0}^{3} I_{2}-2 \sigma_{0}\left(\mu_{1} \rho_{1}+1\right) I_{3}\right], \\
\tilde{a}_{12}= & \frac{2 \alpha^{2}\left(\sigma_{0}+\mu_{2} \rho_{1}\right)}{\sigma_{0} \int_{\Omega} e_{1}^{2} d x}\left[\left(\mu_{1} \rho_{1}+1-\sigma_{0}\right) I_{1}+4 \sigma_{0}^{3} I_{2}+2 \sigma_{0}\left(\mu_{1} \rho_{1}+1\right) I_{3}\right. \\
& \left.+2\left(\mu_{1} \rho_{1}+1\right) J_{1}-8 \sigma_{0}^{2}\left(\mu_{1} \rho_{1}+1\right) J_{2}+4 \sigma_{0}^{2} J_{3}\right] \\
\tilde{b}_{03}= & \frac{2 \alpha^{2}\left(\sigma_{0}-\mu_{2} \rho_{1}\right)}{\sigma_{0} \int_{\Omega} e_{1}^{2} d x}\left[\left(\mu_{1} \rho_{1}+1-\sigma_{0}\right) J_{1}+4 \sigma_{0}^{3} J_{2}+2 \sigma_{0}\left(\mu_{1} \rho_{1}+1\right) J_{3}\right] \\
\tilde{b}_{21}= & \frac{2 \alpha^{2}\left(\sigma_{0}-\mu_{2} \rho_{1}\right)}{\sigma_{0} \int_{\Omega} e_{1}^{2} d x}\left[2\left(\mu_{1} \rho_{1}+1\right) I_{1}-8 \sigma_{0}^{2}\left(\mu_{1} \rho_{1}+1\right) I_{2}+4 \sigma_{0}^{2} I_{3}\right. \\
& \left.+\left(\mu_{1} \rho_{1}+1+\sigma_{0}\right) J_{1}-4 \sigma_{0}^{3} J_{2}-2 \sigma_{0}\left(\mu_{1} \rho_{1}+1\right) J_{3}\right]
\end{aligned}
$$

where

$$
\begin{aligned}
I_{i} & =A_{i}+\sigma_{0}-\int_{\Omega} E_{i} e_{1}^{2} d x \\
J_{i} & =A_{i}-\sigma_{0} \int_{\Omega} E_{i} e_{1}^{2} d x \\
A_{i} & =\left(2 \lambda_{1}-\mu_{2} \rho_{1}-\alpha^{2}\right) \int_{\Omega} E_{i} e_{1}^{2} d x+\alpha^{2} \int_{\Omega} F_{i} e_{1}^{2} d x, \quad i=1,2,3 .
\end{aligned}
$$

Then, the number

$$
\begin{aligned}
b_{1}= & \frac{\pi}{2 \sigma_{0}}\left(a_{02} b_{02}-a_{20} b_{20}\right)+\frac{\pi}{4 \sigma_{0}}\left(a_{11} a_{20}+a_{11} a_{02}-b_{11} b_{20}-b_{11} b_{02}\right) \\
& +\frac{3 \pi}{4}\left(a_{30}+b_{30}+\tilde{a}_{30}+\tilde{b}_{30}\right)+\frac{\pi}{4}\left(a_{12}+b_{21}+\tilde{a}_{12}+\tilde{b}_{21}\right)
\end{aligned}
$$

is the same as in (5.6). Thus Assertions (1)-(2) of this theorem follows from Theorem A.6 in [10].

It is known that the bifurcated periodic solution near $\lambda=\lambda_{1}$ takes the form

$$
v^{\lambda}=x(t) \xi+y(t) \eta+o(|x|+|y|),
$$

where $\xi, \eta$ are as in (5.9) and (5.10), and $(x(t), y(t))$ is the solution of the following equation

$$
\begin{aligned}
& \frac{d x}{d t}=\gamma(\lambda) x-\sigma_{0}(\lambda) y+\frac{1}{\left(\xi_{\lambda}, \xi_{\lambda}^{*}\right)}\left(G\left(x \xi_{\lambda}+y \eta_{\lambda}+\Phi_{\lambda}\right), \xi_{\lambda}^{*}\right) \\
& \frac{d y}{d t}=\sigma_{0}(\lambda) x+\gamma(\lambda) y+\frac{1}{\left(\eta_{\lambda}, \eta_{\lambda}^{*}\right)}\left(G\left(x \xi_{\lambda}+y \eta_{\lambda}+\Phi_{\lambda}\right), \eta_{\lambda}^{*}\right)
\end{aligned}
$$

where $\xi_{\lambda}, \eta_{\lambda}$ are eigenvectors of $L_{\lambda}$ corresponding to the first complex eigenvalues $\beta_{1}^{ \pm}=\gamma \pm i \sigma_{0}$, and $\xi_{\lambda}^{*}, \eta_{\lambda}^{*}$ the conjugate eigenvectors. This solution $(x(t), y(t))$ near 
$\lambda_{1}$ is of the form

$$
\begin{aligned}
& x(t)=\left[-\frac{\gamma(\lambda)}{b_{1}}\right]^{1 / 2} \cos \sigma_{0} t+o\left(|\gamma(\lambda)|^{1 / 2}\right), \\
& y(t)=\left[-\frac{\gamma(\lambda)}{b_{1}}\right]^{1 / 2} \sin \sigma_{0} t+o\left(|\gamma(\lambda)|^{1 / 2}\right),
\end{aligned}
$$

where $b_{1}$ is as in (5.6). Therefore, Assertion (3) follows from (5.20) and (5.21). The proof is complete.

\section{One-Dimensional CASE}

When the containers $\Omega$ are taken as rectangles, the criteria in Theorems 4.2 and 5.2 can be simplified. For simplicity, we consider here only the one-dimensional case:

$$
\Omega=(0, L) \subseteq \mathbb{R}^{1} .
$$

The eigenvalues $\rho_{k}$ and corresponding eigenvectors of (3.2) are given by

$$
\begin{aligned}
& \rho_{k}=\left\{\begin{array}{ll}
k^{2} \pi^{2} / L^{2} & \text { for b.c. (2.4), } \\
(k-1)^{2} \pi^{2} / L^{2} & \text { for b.c. (2.5), }
\end{array} \quad(k=1,2, \cdots),\right. \\
& e_{k}= \begin{cases}\sin \frac{k \pi x}{L} & \text { for b.c. (2.4), } \\
\cos \frac{(k-1) \pi x}{L} & \text { for b.c. (2.5). }\end{cases}
\end{aligned}
$$

Thus the two critical numbers $\lambda_{0}$ and $\lambda_{1}$ in (3.5) and (3.6) are given by

$$
\begin{aligned}
& \lambda_{0}=\min _{k^{2}}\left[\frac{\mu_{1} k^{2} \pi^{2}}{L^{2}}+\frac{\alpha^{2} L^{2}}{\mu_{2} k^{2} \pi^{2}}+\frac{\mu_{1} \alpha^{2}}{\mu_{2}}+1\right], \\
& \lambda_{1}= \begin{cases}\frac{\pi^{2}}{L^{2}}\left(\mu_{1}+\mu_{2}\right)+\alpha^{2}+1 & \text { for b.c. (2.4), } \\
\alpha^{2}+1 & \text { for b.c. (2.5). }\end{cases}
\end{aligned}
$$

It is known that the criterion $b_{1}$ in Theorem 4.2 is valid only for the free boundary condition, which can be expressed explicitly by (4.19). Likewise, for the number defined by (5.6) we have the following explicit expression

$$
b_{1}=\frac{2 \pi \alpha^{2}}{L} b_{0},
$$

with

$$
\begin{aligned}
b_{0}= & \frac{4^{3} \times L}{9 \sigma_{0}^{2} \pi^{2}}\left(\mu_{1} \rho_{1}+1\right)\left(\mu_{2}^{2} \rho_{1}^{2}+2 \mu_{1} \mu_{2} \rho_{1}^{2}+2 \mu_{2} \rho_{1}-\sigma_{0}^{2}\right) \\
& -\frac{3 L}{16}\left(2 \mu_{2} \rho_{1}+3 \alpha^{2}\right)+2\left(3 \mu_{1} \rho_{1}+\mu_{2} \rho_{1}+3\right) A_{1} \\
& -8 \sigma_{0}^{2}\left(\mu_{1} \rho_{1}+\mu_{2} \rho_{1}+1\right) A_{2}-4\left(\mu_{1} \mu_{2} \rho_{1}^{2}+\mu_{2} \rho_{1}-\sigma_{0}^{2}\right) A_{3} \\
& +2\left(\mu_{1} \mu_{2} \rho_{1}^{2}+\mu_{2} \rho_{1}+\sigma_{0}\right) B_{1}+8 \sigma_{0}^{2}\left(\mu_{1} \mu_{2} \rho_{1}^{2}+\mu_{2} \rho_{1}-\sigma_{0}\right) B_{2} \\
& -4 \sigma_{0}^{2}\left(\mu_{1} \rho_{1}+\mu_{2} \rho_{1}+1\right) B_{3},
\end{aligned}
$$


where $A_{i}=\left(2 \mu_{1} \rho_{1}+\mu_{2} \rho_{1}+\alpha^{2}+2\right) B_{i}+\alpha^{2} C_{i}(i=1,2,3)$, and

$$
\begin{aligned}
& B_{1}=32 L \sum_{k=1}^{\infty} \frac{\mu_{2}}{\operatorname{det} M_{2 k+1}\left[(2 k+1)^{2}-4\right]^{2} L^{2}}, \\
& B_{2}=33 L \sum_{k=1}^{\infty} \frac{\left(\mu_{2} \rho_{2 k+1}+\alpha^{2}\right) D_{2 k+1}+\alpha^{2} Q_{2 k+1}}{\operatorname{det} M_{2 k+1} \operatorname{det}\left(M_{2 k+1}^{2}+4 \sigma_{0}^{2}\right) \pi^{2}(2 k+1)^{2}\left[(2 k+1)^{2}-4\right]^{2}} \\
& B_{3}=32 L \sum_{k=1}^{\infty} \frac{D_{2 k+1}}{\operatorname{det}\left(M_{2 k+1}^{2}+4 \sigma_{0}^{2}\right) \pi^{2}(2 k+1)^{2}\left[(2 k+1)^{2}-4\right]^{2}}, \\
& C_{1}=-32 L \sum_{k=1}^{\infty} \frac{\mu_{1} \rho_{1}+1}{\operatorname{det} M_{2 k+1} \pi^{2}(2 k+1)^{2}\left[(2 k+1)^{2}-4\right]^{2}}, \\
& C_{2}=-32 L \sum_{k=1}^{\infty} \frac{\left(\mu_{1} \rho_{1}+\mu_{2} \rho_{1}+\alpha^{2}+1, D_{2 k+1}-\left(\mu_{1} \rho_{k}-\mu_{1} \rho_{1}-\mu_{2} \rho_{1}-\alpha^{2}\right) Q_{2 k+1}\right.}{\left.\operatorname{det} M_{2 k+1} \operatorname{det}\left(M_{2 k+1}^{2}+4 \sigma_{0}^{2}\right) \pi^{2}(2 k+1)^{2}\left[(2 k+1)^{2}-4\right]^{2}\right]} \\
& C_{3}=32 L \sum_{k=1}^{\infty} \frac{Q_{2 k+1}}{\operatorname{det}\left(M_{2 k+1}^{2}+4 \sigma_{0}^{2}\right) \pi^{2}(2 k+1)^{2}\left[(2 k+1)^{2}-4\right]^{2}} .
\end{aligned}
$$

Let $\lambda_{0}$ in (6.4) achieves its minimum at the integer $k_{0}^{2}$, i.e.,

$$
\lambda_{0}=\frac{\mu_{1} k_{0}^{2} \pi^{2}}{L^{2}}+\frac{\alpha^{2} L^{2}}{\mu_{2} k_{0}^{2} \pi^{2}}+\frac{\mu_{1}}{\mu_{2}} \alpha^{2}+1 .
$$

Then $k_{0} \geq 1$ satisfies

$$
k_{0}\left(k_{0}-1\right) \leq \frac{\alpha L^{2}}{\pi^{2} \sqrt{\mu_{1} \mu_{2}}} \leq k_{0}\left(k_{0}+1\right) .
$$

To see this, note that the function

$$
\lambda(x)=\frac{\mu_{1} \pi^{2} x}{L^{2}}+\frac{\alpha^{2} L^{2}}{\mu_{2} \pi^{2} x}+\frac{\mu_{1}}{\mu_{2}} \alpha^{2}+1
$$

has its minimum at $x_{0}=\alpha L^{2} / \pi^{2} \sqrt{\mu_{1} \mu_{2}}$, and

$$
\frac{d \lambda}{d x} \begin{cases}<0 & \text { if } x<x_{0}, \\ >0 & \text { if } x>x_{0} .\end{cases}
$$

It follows that either $k_{0}=m$ or $k_{0}=m+1$, such that $m=\sqrt{x_{0}}-\varepsilon$ for some $0<\varepsilon<1$; namely

$$
m^{2} \leq x_{0} \leq(m+1)^{2} .
$$

It follows that

$$
k_{0}= \begin{cases}m & \text { if } \lambda\left(m^{2}\right)<\lambda\left((m+1)^{2}\right), \\ m \text { and } m+1 & \text { if } \lambda\left(m^{2}\right)=\lambda\left((m+1)^{2}\right), \\ m+1 & \text { if } \lambda\left(m^{2}\right)>\lambda\left((m+1)^{2}\right) .\end{cases}
$$

We infer from (6.10) that

$$
\begin{aligned}
& \frac{\alpha L^{2}}{\pi^{2} \sqrt{\mu_{1} \mu_{2}}} \leq m(m+1) \Rightarrow k_{0}=m, \\
& \frac{\alpha L^{2}}{\pi^{2} \sqrt{\mu_{1} \mu_{2}}} \geq m(m+1) \Rightarrow k_{0}=m+1,
\end{aligned}
$$


which yield the inequalities (6.9).

In the following, we compare $\lambda_{0}$ with $\lambda_{1}$ in terms of the parameters $\mu_{1}, \mu_{2}, \alpha$, and $L$. We proceed in two cases.

The CASE WHERE $\mu_{1} \geq \mu_{2}$ : Then, from (3.5)-(3.6), we see that

$$
\lambda_{1}<\lambda_{0} \quad \forall L, \alpha>0 \text { for b.c. (2.5). }
$$

For b.c. (2.4), we can prove that

$$
\begin{aligned}
& \lambda_{0}>\lambda_{1} \\
& \lambda_{1}<\lambda_{0}, \quad \text { if } L>L_{c},
\end{aligned} \quad \text { if } 0<L<L_{c},
$$

where

$$
L_{c}^{2}=\frac{\pi^{2}}{2 \alpha}\left[\sqrt{\left(\mu_{1}-\mu_{2}\right)^{2} \alpha^{2}+4 \mu_{2}^{2}}-\alpha\left(\mu_{1}-\mu_{2}\right)\right] .
$$

In fact, from $\lambda_{0}=\lambda_{1}$, we derive the critical scale $L_{c}$ as

$$
L_{c}^{2}=\frac{k_{0}^{2} \pi^{2}}{2 \alpha}\left[\sqrt{\left(\mu_{1}-\mu_{2}\right)^{2} \alpha^{2}+\frac{4 \mu_{2}}{k_{0}^{2}}\left(\mu_{1}+\mu_{2}-k_{0}^{2} \mu_{1}\right)}-\alpha\left(\mu_{1}-\mu_{2}\right)\right],
$$

provided that

$$
\mu_{1}+\mu_{2}>k_{0}^{2} \mu_{1}
$$

Since $\mu_{1} \geq \mu_{2}$, it implies that $k_{0}=1$. Hence $L_{c}$ is as in (6.13), and (6.12) holds true.

We remark that by (6.9), $L_{c}^{2}$ in (6.13) has to satisfy the inequality

$$
L_{c}^{2} \leq 2 \pi^{2} \sqrt{\mu_{1} \mu_{2}} / \alpha,
$$

which holds true for $\mu_{1} \geq \mu_{2}$.

The CASE WHERE $\mu_{1}<\mu_{2}$ : For b.c. (2.4), we deduce from $\lambda_{0}=\lambda_{1}$ the following two critical scales:

$$
L_{c_{1,2}}^{2}=\frac{k_{0}^{2} \pi^{2}}{2 \alpha}\left[\left(\mu_{2}-\mu_{1}\right) \alpha \mp \sqrt{\left(\mu_{2}-\mu_{1}\right)^{2} \alpha^{2}-\frac{4 \mu_{2}}{k_{0}^{2}}\left(k_{0}^{2} \mu_{1}-\mu_{1}-\mu_{2}\right)}\right],
$$

with $L_{c_{1}}^{2}<L_{c_{2}}^{2}$.

It is easy to see that for the boundary condition (2.4),

$$
\lambda_{1}<\lambda_{0} \quad \text { if }\left\{\begin{array}{l}
k_{0}^{2} \alpha^{2}\left(\mu_{2}-\mu_{1}\right)^{2}<4 \mu_{2}\left(k_{0}^{2} \mu_{1}-\mu_{1}-\mu_{2}\right), \\
\text { or } \\
k_{0}^{2} \alpha^{2}\left(\mu_{2}-\mu_{1}\right)^{2}>4 \mu_{2}\left(k_{0}^{2} \mu_{1}-\mu_{1}-\mu_{2}\right) \text { and } \\
0<L^{2}<L_{c_{1}}^{2} \text { or } L_{c_{2}}^{2}<L^{2},
\end{array}\right.
$$

$$
\lambda_{0}<\lambda_{1} \quad \text { if } \begin{cases}L_{c_{1}}^{2}<L^{2}<L_{c_{2}}^{2} & \text { for } 0<4 \mu_{2}\left(k_{0}^{2} \mu_{1}-\mu_{1} \mu_{2}\right)<k_{0}^{2} \alpha^{2}\left(\mu_{2}-\mu_{1}\right), \\ \text { or } 0<L^{2}<L_{c_{2}}^{2} & \text { for } k_{0}^{2} \mu_{1} \leq \mu_{1}+\mu_{2} .\end{cases}
$$

For the boundary condition (2.5), we obtain two critical scales as

$$
l_{c_{1,2}}^{2}=\frac{k_{0}^{2} \pi^{2}}{2 \alpha}\left[\left(\mu_{2}-\mu_{1}\right) \alpha \mp \sqrt{\left(\mu_{2}-\mu_{1}\right)^{2} \alpha^{2}-4 \mu_{1} \mu_{2}}\right],
$$


such that

$$
\begin{aligned}
& \lambda_{1}<\lambda_{0} \text { if }\left\{\begin{array}{l}
\left(\mu_{2}-\mu_{1}\right)^{2} \alpha^{2}<4 \mu_{1} \mu_{2}, \\
\text { or } 0<L^{2}<l_{c_{1}}^{2} \text { or } l_{c_{2}}^{2}<L^{2} \\
\text { for }\left(\mu_{2}-\mu_{1}\right)^{2} \alpha^{2}>4 \mu_{1} \mu_{2}
\end{array}\right. \\
& \lambda_{0}<\lambda_{1} \text { for b.c. (2.5) if } l_{c_{1}}^{2}<L^{2}<l_{c_{2}}^{2} \text { for }\left(\mu_{2}-\mu_{1}\right)^{2} \alpha^{2}>4 \mu_{1} \mu_{2} .
\end{aligned}
$$

\section{Physical REMARKS}

We now discuss the phase transition of Brusselator by using Theorem 4.1 15.2 for the one-dimensional case 6.1).

Dirichlet Boundary Condition. When $\mu_{1} \geq \mu_{2}$, by (6.12), the system (2.3)-(2.4) has a transition to steady states provided $0<L<L_{c}$, and to periodic solutions provided $L_{c}<L$.

Physical Conclusion 7.1. Let $\mu_{1} \geq \mu_{2}$. Then for the system (2.3)- 2.4), we have the following conclusions:

(1) When $0<L<L_{c}$, the transition of (2.3)- 2.4) at $\lambda=\lambda_{0}$ is of Type-III, and there is a saddle-node bifurcation at some $0<\lambda^{*}<\lambda_{0}$. In other wards, the basic state $u_{0}=(\alpha, \lambda / \alpha)$ is stable for $0<\lambda<\lambda^{*}$, is metastable for $\lambda^{*}<\lambda<\lambda_{0}$, and is unstable for $\lambda^{*}<\lambda$. Moreover, if $\lambda^{*}<\lambda$, there are at least two metastable equilibrium states.

(2) When $L_{c}<L$, this system undergoes a dynamic transition at $\lambda=\lambda_{1}$ to periodic solutions. In particular, there exists an $L_{0}>L_{c}$ such that if $L_{c}<L<L_{0}$, the transition is of Type-II, and there is a singular separation of periodic solutions at some $\tilde{\lambda}<\lambda_{1}$. If $L_{0}<L$, the transition is of Type-I, and the system bifurcates from $u_{0}$ to a stable periodic solution on $\lambda>\lambda_{1}$, which is expressed as $u_{\lambda}=\left(u_{1}^{\lambda}, u_{2}^{\lambda}\right)$ with

$$
\begin{aligned}
& u_{1}^{\lambda}=\alpha+2 \alpha^{2} \sqrt{\frac{\lambda-\lambda_{1}}{\left|b_{1}\right|}} \sin \frac{\pi x}{L} \sin \left(\sigma_{0} t+\frac{\pi}{4}\right)+o\left(\left|\lambda-\lambda_{1}\right|^{1 / 2}\right), \\
& u_{2}^{\lambda}=\frac{\lambda}{\alpha}+2\left(\sigma_{0}^{2}+\left(\alpha^{2}+\frac{\mu \pi^{2}}{L^{2}}\right)^{2}\right) \sqrt{\frac{\lambda-\lambda_{1}}{\left|b_{1}\right|}} \sin \frac{\pi x}{L} \cos \left(\sigma_{0} t+\theta\right)+o\left(\left|\lambda-\lambda_{1}\right|^{1 / 2}\right) .
\end{aligned}
$$

This periodic solution provides a spatial-temporal oscillation of the Brusselator.

The first conclusion is due to Theorem 4.1 the existence of global attractors, and the fact that $u_{0}=(0,0)$ is a unique steady state solution of (2.3)-(2.4) at $\lambda=0$.

The second conclusion is based on Theorem 5.2 and the following analysis on the criterion $b_{0}$ given by (6.7). We know that

$$
\lambda_{1}-\lambda_{0} \rightarrow 0 \quad \text { as } \quad L \rightarrow L_{c},
$$

which implies

$$
\sigma_{0} \rightarrow 0 \quad \text { as } \quad L \rightarrow L_{c} .
$$

It follows from (6.7) that $b_{0} \rightarrow+\infty$ for $L \rightarrow L_{c}+0$. Therefore

$$
b_{0}>0 \quad \forall L_{c}<L<L_{0},
$$


for some $L_{0}>L_{c}$. On the other hand, $\rho_{k} \rightarrow 0(L \rightarrow \infty)$. Hence, when $L \rightarrow \infty$,

$$
\begin{aligned}
b_{0} \rightarrow & -\frac{64 L}{9 \pi^{2}}-\frac{9 \alpha^{2} L}{16}+2\left(3 \alpha^{2}+6+\sigma_{0}\right) B_{1}-8 \sigma_{0}^{2}\left(\alpha^{2}+\sigma_{0}+2\right) B_{2} \\
& +4 \alpha^{2}\left(\alpha^{2}+1\right) B_{3}+6 \alpha^{2} C_{1}-8 \sigma_{0}^{2} \alpha^{2} C_{2}+4 \alpha^{4} C_{3} .
\end{aligned}
$$

Note that

$$
\begin{array}{lll}
\sigma_{0} \rightarrow \alpha, & D_{k} \rightarrow 3 \alpha^{2}, & Q_{k} \rightarrow-3 \alpha^{2}, \\
\operatorname{det} M_{k} \rightarrow \alpha^{2}, & \operatorname{det}\left(M_{k}^{2}+4 \sigma_{0}^{2}\right) \rightarrow 9 \alpha^{4}, & B_{1} \rightarrow 0, \\
B_{2} \rightarrow 0, & B_{3} \rightarrow \frac{32 L}{3 \alpha^{2}} E, & C_{1} \rightarrow-\frac{32 L}{\alpha^{2}} E, \\
C_{2} \rightarrow-\frac{32 L}{3 \alpha^{4}} E, & C_{3} \rightarrow-\frac{32 L}{3 \alpha^{2}} E, &
\end{array}
$$

for $L \rightarrow \infty$, where

$$
E=\sum_{k=1}^{\infty} \frac{1}{\pi^{2}(2 k+1)^{2}\left[(2 k+1)^{2}-4\right]^{2}} .
$$

Thus, in view of (7.2),

$$
b_{0} \rightarrow-L\left[\frac{64}{9 \pi^{2}}+\frac{9 \alpha^{2}}{16}+64 E\right]<0 \quad \text { as } L \rightarrow \infty .
$$

Hence

$$
b_{0}<0 \quad \forall L_{1}<L
$$

for some $L_{1} \geq L_{0}$.

From the physical point of view, it is reasonable to consider the case where $b_{0}$ changes its sign only once in $\left(L_{c}, \infty\right)$. Hence, physically, we have $L_{0}=L_{1}$. Thus, we derive from (7.1) and (7.3) the second conclusion.

Now, we consider the case where $\mu_{1}<\mu_{2}$ by the following two examples. We take

$$
\mu_{1}=2 \times 10^{-3}, \quad \mu_{2}=4 \times 10^{-3} .
$$

Example 7.1. Let (7.4) hold true, and $\alpha=2, L=4$. Then we obtain from (6.9) that $k_{0}=34$, and

$$
k_{0}^{2} \alpha^{2}\left(\mu_{2}-\mu_{1}\right)^{2}<4 \mu_{2}\left(k_{0}^{2} \mu_{1}-\mu_{1}-\mu_{2}\right) .
$$

In view of (6.15),

$$
\lambda_{1}<\lambda_{0} \text { for b.c. (2.4). }
$$

The number $b_{0}$ in (6.7) is given by

$$
b_{0} \cong-\left(\frac{64}{9 \pi^{2}}+\frac{9}{4}\right) L+40 B_{1}-256 B_{2}+80 B_{3}+24 C_{1}-128 C_{2}+64 C_{3},
$$

and

$$
\begin{array}{ll}
B_{1} \cong \frac{2}{25} \times 10^{-3} L, & B_{2} \cong-\frac{11}{36 \times 25} \times 10^{-3} L, \\
B_{3} \cong \frac{8}{27 \times 25 \pi^{2}} L, & C_{1} \cong-\frac{8}{9 \times 25 \pi^{2}} L, \\
C_{2} \cong-\frac{2}{27 \times 25 \pi^{2}} L, & C_{3} \cong-\frac{8}{27 \times 25 \pi^{2}} L .
\end{array}
$$


It is easy to see that $b_{0}<0$. Then, by Theorem 5.2, the phase transition of (2.3) $-(2.4)$ is of Type-I, and this system undergoes a spatial-temporal oscillation on $\lambda>\lambda_{1}$.

Example 7.2. Assume (7.4) and $\alpha=3, L=4$. Then $k_{0}=41$, and

$$
k_{0}^{2} \alpha^{2}\left(\mu_{2}-\mu_{1}\right)^{2}>4 \mu_{2}\left(k_{0}^{2} \mu_{1}-\mu_{1}-\mu_{2}\right)>0 .
$$

Thus, we can obtain two critical scales $L_{c_{1}}^{2}$ and $L_{c_{2}}^{2}$ in (6.14) as follows:

$$
L_{c_{1}}^{2}=11.07, \quad L_{c_{2}}^{2}=22.14 \text {. }
$$

Hence

$$
L_{c_{1}}^{2}<L^{2}=16<L_{c_{2}}^{2}
$$

which leads (by (6.16)) to

$$
\lambda_{0}<\lambda_{1} \quad \text { for b.c. (2.4). }
$$

It is clear that

$$
\int_{\Omega} e_{k_{0}}^{3} d x=\int_{0}^{L} \sin ^{3} \frac{k_{0} \pi x}{L} d x \neq 0
$$

By (7.5) and Theorem 4.1, the system (2.3) and (2.4) has a Type-III transition at $\lambda=\lambda_{0}$, and there is a saddle-node bifurcation at some $\lambda^{*}\left(0<\lambda^{*}<\lambda_{0}\right)$.

Neumann Boundary Condition. Consider the case where $\mu_{1} \geq \mu_{2}$. Based on (6.15) and Theorem 5.1] we have the following physical conclusion.

Physical Conclusion 7.2. Let $\mu_{1} \geq \mu_{2}$. Then the system (2.3) with (2.5) has a Type-I transition to periodic solutions at $\lambda=\lambda_{1}$, i.e., a spatial-temporal oscillation occurs in the Brusselator for $\lambda>\lambda_{1}$.

For the case where $\mu_{1}<\mu_{2}$, we have the following example.

Example 7.3. Under the same conditions as in Example 7.2, $k_{0}=41$ and the two critical scales $l_{c_{1}}^{2}$ and $l_{c_{2}}^{2}$ in (6.17) are given by

$$
l_{c_{1}}^{2}=11.06, \quad l_{c_{2}}^{2}=22.12 .
$$

Hence, $l_{c_{1}}^{2}<l^{2}=16<l_{c_{2}}^{2}$, which implies, by (6.19), that

$$
\lambda_{0}<\lambda_{1} \quad \text { for b.c. (2.5). }
$$

On the other hand, by (6.2) and (6.3), we have

$$
\rho_{k_{0}+1}=\frac{k_{0}^{2} \pi^{2}}{L^{2}} \cong 10^{3}, \quad \int_{\Omega} e_{k_{0}+1}^{3} d x=\int_{0}^{L} \cos ^{3} \frac{k_{0} \pi x}{L} d x=0 .
$$

Thus, it is easy to check that the number $b_{1}$ in (4.10), which is also given by (4.19), is negative, i.e.,

$$
b_{1}<0 \quad \text { in (4.10). }
$$

By (7.6)-(7.7) and Theorem 4.2, the system (2.3) with (2.5) bifurcates on $\lambda>\lambda_{0}$ to two stable steady states $v_{ \pm}^{\lambda}$ as given by (4.12). It shows that the Brusselator undergoes a transition at $\lambda_{0}=9.8$. 


\section{REFERENCES}

[1] W. BAO, The random projection method for a model problem of combustion with stiff chemical reactions, Appl. Math. Comput., 130 (2002), pp. 561-571.

[2] B. Guo And Y. Han, Attractor and spatial chaos for the Brusselator in $\mathbf{R}^{N}$, Nonlinear Anal., 70 (2009), pp. 3917-3931.

[3] C.-H. Hsia, T. MA, And S. WANG, Rotating boussinesq equations: Dynamic stability and transitions, DCDS-A, 28:1 (2010), pp. 99-130.

[4] H. G. Kaper And T. J. Kaper, Asymptotic analysis of two reduction methods for systems of chemical reactions, Phys. D, 165 (2002), pp. 66-93.

[5] T. Ma And S. Wang, Phase Transition Dynamics in Nonlinear Sciences, submitted.

[6] — Bifurcation theory and applications, vol. 53 of World Scientific Series on Nonlinear Science. Series A: Monographs and Treatises, World Scientific Publishing Co. Pte. Ltd., Hackensack, NJ, 2005.

[7] — Stability and Bifurcation of Nonlinear Evolutions Equations, Science Press, 2007.

[8] - Dynamic phase transition theory in PVT systems, Indiana University Mathematics Journal,, 57:6 (2008), pp. 2861-2889.

[9] — Dynamic transition theory for thermohaline circulation, Physica D, 239 (2009), pp. $167-189$.

[10] - Tropical atmospheric circulations: Dynamic stability and transitions, Discrete and Continuous Dynamical Systems, Ser. A, 26:4 (2009), pp. 1399-1417.

[11] I. Prigogine and R. Lefever, Symmetry breaking instabilities in dissipative systems, II. J. Chem. Phys., 48 (1968), p. 1695.

[12] J. SHI, Bifurcation in infinite dimensional spaces and applications in spatiotemporal biological and chemical models, Front. Math. China, 4 (2009), pp. 407-424.

[13] J. C. Tzou, B. J. Matkowsky, and V. A. Volpert, Interaction of Turing and Hopf modes in the superdiffusive Brusselator model, Appl. Math. Lett., 22 (2009), pp. 1432-1437.

(TM) Department of Mathematics, Sichuan University, Chengdu, P. R. China

(SW) Department of Mathematics, Indiana University, Bloomington, IN 47405

E-mail address: showang@indiana.edu, http://www.indiana.edu/ ffluid 